This is an author produced version of a paper published in Remote Sensing of Environment. This paper has been peer-reviewed but does not include the final publisher proof-corrections or journal pagination.

Citation for the published paper:

Jamali, Sadegh; Jönsson, Per; Eklundh, Lars; Ardö, Jonas; Seaquist, Jonathan. (2015). Detecting changes in vegetation trends using time series segmentation. Remote Sensing of Environment, vol. 156, p. null

URL: https://doi.org/10.1016/j.rse.2014.09.010

Publisher: Elsevier

This document has been downloaded from MUEP (https://muep.mah.se) / DIVA (https://mau.diva-portal.org). 


\title{
Detecting Changes in Vegetation Trends using Time Series Segmentation
}

Sadegh Jamali ${ }^{\text {a, } *}$, Per Jönsson ${ }^{\mathrm{b}}$, Lars Eklundh ${ }^{\mathrm{a}}$, Jonas Ardö ${ }^{\mathrm{a}}$, Jonathan Seaquist ${ }^{\mathrm{a}}$

\begin{abstract}
Affiliations:
\end{abstract}
${ }^{\text {a }}$ Department of Physical Geography and Ecosystem Science,

Lund University, Sölvegatan 12, SE-223 62 Lund, Sweden

${ }^{\mathrm{b}}$ Division of Materials Science and Applied Mathematics,

Malmö University, Sweden

* Corresponding author:

Sadegh Jamali

Department of Physical Geography and Ecosystem Science

Lund University

Sölvegatan 12

SE-223 62 Lund

Sweden

Phone: +46 462221725

Fax: +46 462220321 
E-mail: Sadegh.Jamali@ nateko.lu.se

Keywords:

Vegetation dynamics, Change detection, Vegetation imagery, Trend analysis, Time series, Segmentation, DBEST, GIMMS, NDVI

\section{Abstract}

Although satellite-based sensors have made vegetation data series available for several decades, the detection of vegetation trend and change is not yet straightforward. This is mainly due to the quality of the available change detection algorithms, which seldom meet users' main need for identifying and characterizing both abrupt and non-abrupt changes, without sacrificing accuracy or computational speed. We propose a user-friendly program for analysing vegetation time series, with two main application domains: generalising vegetation trends to main features, and characterizing vegetation trend changes. This program, Detecting Breakpoints and Estimating Segments in Trend (DBEST) uses a novel segmentation algorithm which simplifies the trend into linear segments using one of three user-defined parameters: a generalisation-threshold parameter $\delta$, the $m$ largest changes, or a threshold $\beta$ for the magnitude of changes of interest for detection. The outputs of DBEST are the simplified trend, the change type (abrupt or non-abrupt), and estimates for the characteristics (time and magnitude) of the change. DBEST was tested and evaluated using simulated Normalized Difference Vegetation Index (NDVI) data at two sites, which included different types of changes. Evaluation results demonstrate that DBEST quickly 
and robustly detects both abrupt and non-abrupt changes, and accurately estimates change time and magnitude.

DBEST was also tested using data from the Global Inventory Modeling and Mapping Studies (GIMMS) NDVI image time series for Iraq for the period 1982-2006, and was able to detect and quantify major change over the area. This showed that DBEST is able to detect and characterize changes over large areas. We conclude that DBEST is a fast, accurate and flexible tool for trend detection, and is applicable to global change studies using time series of remotely sensed data sets.

\section{Introduction}

Vegetation is an important component of terrestrial ecosystems (e.g. Prentice et al., 2000; Sitch et al., 2003). It is now widely recognized that vegetation can respond to climate and disturbance in a complex, non-linear fashion (e.g. Lambin and Ehrlich, 1997). External disturbances to ecosystems, including insect outbreaks, fires, forest clear-cutting, and conversion of natural grasslands to cultivated crops, increase the complexity of vegetation variations across a wide range of spatio-temporal resolutions and scales (e.g. Higgins et al., 2002; Lambin et al., 2003). There is a corresponding need for data and tools which can properly capture and represent such complex dynamics, at a level of detail which is appropriate to the question under consideration. One flexible and simplifying idea is to allow the user to define the boundary between signal and 
noise. Developing analysis tools that embody this idea can help us to characterize and understand vegetation dynamics with much greater efficiency.

Vegetation dynamics are often monitored at different spatial and temporal scales through vegetation index data series, such as GIMMS (Global Inventory Modeling and Mapping Studies), MODIS (Moderate Resolution Imaging Spectroradiometer), and SPOT/Vegetation (System Pour l'Observation de la Terre) data sets. One widely used index is the Normalized Difference Vegetation Index (NDVI), which is an indicator of the vegetation's greenness (Rouse et al., 1973). Temporal trend analysis of NDVI has proved particularly useful for monitoring and characterizing the response of land cover to phenomena with a range of time scales, from abrupt natural or anthropogenic events (Verbesselt et al., 2009), to seasonal variability of plant phenology caused by changes in temperature and rainfall regimes (Heumann et al., 2007), through to gradual inter-annual climate changes (Jacquin et al., 2010). Similar analyses of NDVI trends have also been used to investigate the relationship between NDVI and Leaf Area Index (LAI), a key variable which is functionally related to plant biomass production. Studies have indicated that this relationship is not completely linear, particularly for high LAI values (e.g. Carlson and Ripley, 1997; Fan et al., 2009).

Non-linear temporal trends in NDVI can be studied by a number of techniques, including time series segmentation (Keogh et al., 2004). Series segmentation refers to approximating a time series by a set of piecewise straight-line segments, in the process eliminating noise but preserving the salient details of the trend. Two significant merits of segmentation are that first, it approximates complex signatures to extract their basic features (Zeileis et al., 2003) and second, it increases the efficiency of data storage and further computation (Keogh et al., 2004). The size 
of the output set can vary between one and the size of the original time series, and is a key parameter in determining the type of information available to the user, from most generalised/compressed information (one segment) to all possible details (all data points).

A particular type of segmentation technique, which always outputs one segment, is the ordinary least squares (OLS) technique. It has been widely used for assessing long-duration changes in vegetation greenness using NDVI time series (Fuller, 1998; Eklundh and Olsson, 2003; Nemani et al., 2003; Fensholt et al., 2009; Peng et al., 2012). However, the implicit assumption that vegetation always changes gradually and constantly over the entire study period is a critical drawback of this method (e.g. Jamali et al., 2014). Consequently, it is impossible using OLS to identify periods in which the rate of change, or even the sign of the rate of change, varies within a given time period: for example, the existence of short-duration nonlinearities such as 'greening' or 'browning' patterns may be completely obscured because they have been averaged out altogether (de Jong et al., 2012). On the other hand, using the original time series in further analysis is not cost effective and may also, in cases when only the main features of the vegetation change are of interest, carry unused detailed information and noise.

Fortunately, to date at least two alternative approaches between the two mentioned extreme cases have been introduced for detecting trends and estimating changes in vegetation time series. The first approach, Landsat-based Detection of Trends in Disturbance and Recovery (LandTrendR), uses arbitrary temporal segmentation to divide long-term trends into piecewise linear segments for the representation of spectral change in forested ecosystems (Cohen et al., 2010; Kennedy et al., 2010). LandTrendR focuses solely on Landsat derived data, and requires several complex control parameters to be specified. Moreover, different signal to noise ratios and spectral 
properties of different instruments may require alteration of the algorithms and parameters used in LandTrendR, and its generalisation for use with time series of other sensors in other ecosystems has not yet been implemented.

The second approach, Breaks For Additive and Seasonal Trend (BFAST), was one of the first general methods for detecting changes in time series data, focusing on the trend and seasonal components in long term NDVI data series, at spatial scales ranging from continental to global (e.g. Verbesselt et al., 2010a; Verbesselt et al., 2010b; de Jong et al., 2012; de Jong et al., 2013). It employs a generic approach by assuming that a time series can be modelled in terms of its trend, seasonal, and remainder components, including breaks in the trend and seasonal components. In BFAST, nonlinearity in the trend component is also simplified into a number of individual trend segments, in order to identify sudden structural shifts. The simplified trend is therefore composed of segments with gradual changes, separated from each other by relatively brief, abrupt changes (Verbesselt et al., 2010a; de Jong et al., 2012; de Jong et al., 2013). Our experience using BFAST (most recent version 1.4.4) shows that although the user can control the number of breaks to be detected, they do not have full control over the level of trend generalisation (whether basic features or detailed information will be extracted), or over the change magnitude that is detected. More importantly, the requirement that gradual changes are separated from each other by a sudden change (most often with a duration of one time step) may not always guarantee the best approximation of trend nonlinearity for real-world data, where changes of different types and durations may exist. 
Thus, to the best of our knowledge, no attention has yet been paid to segmenting time series of vegetation indices to generalise trends and detect both abrupt and non-abrupt changes, in a way which allows the user to control the segmentation. Ideally the user should be able to choose the generalisation scheme and extract information about both the main feature and details. The user should also be able to detect and characterize changes of interest, including realistic abrupt and non-abrupt changes of different durations and in any order within a particular sequence of occurrences.

The overall aim of this paper is to propose a novel approach for segmenting and analysing trend changes in time series of vegetation indices (VI). The method, called Detecting Breakpoints and Estimating Segments in Trend (DBEST), provides both generalised trend segments and estimates of the characteristics of both abrupt events and long-term processes. DBEST has been tested and evaluated using simulated NDVI data series. We also applied DBEST to a GIMMS-NDVI dataset (1982-2006) for Iraq, to detect major changes, determine change type (abrupt or nonabrupt) and estimate the characteristics (time, magnitude) of the changes in a spatio-temporal framework. The method allows for fast, flexible and accurate estimations of trends, essential from a global change perspective.

\section{Data and study area}

We have developed our method using a commonly used time-series data source: the NASA GIMMS data set. Then, we applied the methods to selected study areas that highlight certain 
temporal features. The methods are not specific to either the data type or the sites, which merely represent commonly used data and illustrative cases.

\subsection{GIMMS data}

The GIMMS data set is a twice-monthly composite NDVI product with global coverage at an 8$\mathrm{km}$ spatial resolution, which is available for the 25.5 year period from July 1981 to December 2006 (Tucker et al., 2004; Tucker et al., 2005). The GIMMS data were derived from the output of the AVHRR (Advanced Very High Resolution Radiometer) instrument on board NOAA (National Oceanographic and Atmospheric Administration) satellite series 7, 9, 11, 14, 16 and 17. The NDVI is computed from the red and near infrared bands of the AVHRR sensor, and is correlated with photosynthetic activity and the LAI of green vegetation. The GIMMS data have been corrected for variations arising from calibration, view geometry, volcanic aerosols, and other factors not related to actual vegetation change (Tucker et al., 2005). The GIMMS data have also been validated against the well-calibrated and atmospherically-corrected MODIS data set for the period 2000-2007, and for the Earth's semi-arid regions (Fensholt et al., 2012). Despite the corrections, the GIMMS data may contain residual invalid measurements, but these are well indicated using quality flags (de Jong et al., 2012). Based on the quality flags, we only considered pixels with at least $75 \%$ valid data points (i.e., flag value zero) through the whole time span.

\subsection{Study area}


The case study was Iraq, selected due to the fact that large parts of the county's land surface have undergone major changes in the recent past. Iraq's geography consists of four main zones: desert in the west and southwest, rolling upland between the upper Tigris and Euphrates rivers in north central Iraq, highlands in the north and northeast with mountains, and alluvial plain through which the Tigris and Euphrates flow from northwest to southeast. About 77.7\% of Iraq's land area is not viable for agriculture. $0.3 \%$ is forest and woodlands situated along the extreme northern border with Turkey and Iran. The remaining $22 \%$ are used for a range of agricultural activities (Schnepf, 2004).

Over the last two to three decades, the landscape of Iraq has witnessed many changes (UNEP, 2001). These have been caused by frequent natural hazards, such as sand storms (Draxler et al., 2001) and floods (Hamdan et al., 2010), and human activities, such as forced migration, social conflicts, and wars (especially in south-eastern and northern Iraq). The high probability of abrupt vegetation disturbances and recoveries made Iraq the ideal study area to test DBEST.

\section{Methods}

In this section, DBEST algorithm is introduced, and then approaches used for validating and evaluating DBEST's performance are explained. The DBEST algorithm consists of two main parts: trend estimation and trend segmentation.

\subsection{The trend estimation method}


For a given VI time series (including seasonal variations), the temporal trend is derived using the Seasonal-Trend decomposition procedure based on Loess (STL) (Cleveland et al., 1990), implemented in an unpublished version of the TIMESAT software package (Jönsson and Eklundh, 2004). STL is a fast filtering procedure able to deal with missing values, for decomposing a time series into trend (low frequency variation), seasonal (variation at or near the seasonal frequency), and remainder (remaining variation) components. TIMESAT is a software package for analysing time series of satellite sensor data, which makes it possible to investigate the seasonality of the data and their relationship with dynamic properties of vegetation such as phenology.

Before using STL, the existence of significant discontinuities in the VI level is examined. To do this, the absolute difference in VI between each pair of consecutive data points, starting from the time series' start point, is compared with a user-defined first level-shift-threshold $\left(\theta_{1}\right)$. If the absolute difference is greater than the threshold value, a second criterion tests whether the change led to a significant shift in the mean VI level which persists throughout a user-defined time period, the duration-threshold $(\phi)$. The second criterion is valid if the absolute difference in the means of the VI data, calculated over a period $\phi$ before and after the current data point, is greater than a user-defined second level-shift-threshold $\left(\theta_{2}\right)$.

If both tests are valid, the current data point is marked as a candidate level-shift point. The same procedure is repeated for every data point in the time series. The candidate points are then sorted into descending order according to the absolute value of the shift in the VI level. The first point 
in the sorted list is marked as the most significant level-shift point. For the second and subsequent candidate points, in addition to the two mentioned criteria, a third criterion should be fulfilled in order to be marked as the next significant level-shift points. The third criterion checks if the spacing between the candidate point and any previously detected level-shift point is at least the duration-threshold $\phi$. Bai and Perron (2003) and Zeileis et al. (2003) also recommend a fraction of data needed between successive breaks. At any detected level-shift point, the VI time series is divided into two parts, before and after.

The STL decomposition is then performed separately for each part of the time series divided by the detected level-shift points (called hereafter separate STL decomposition) if one or more level-shift points have been detected: if no such points are found, it is performed once for the entire time series. The separate STL decomposition procedure often results in more precise trend and seasonal components (especially around the detected level-shift points) because the shifts in the VI level are not deformed by the smoothing methods used in the STL method (Cleveland et al., 1990).

If the given time series is already deseasonalized (i.e. we are examining the trend component) then significant level-shifts are similarly detected but of course no STL decomposing is needed.

\subsection{The segmentation method}

For the trend time series (either computed using the STL or already deseasonalized), with a number of observations $N(N>2)$, single time-step differences in the forward and backward 
directions are calculated at every time-point $i(2 \leq i \leq N-1)$, as follows (Fig. 1a):

$$
\begin{aligned}
& \Delta V I_{(i-1, i)}=\left(V I_{(i)}-V I_{(i-1)}\right) \\
& \Delta V I_{(i, i+1)}=\left(V I_{(i+1)}-V I_{(i)}\right)
\end{aligned}
$$

For each point $i$ the peak/valley detector function $(f)$ is then computed, based on the continuity of the sign of the two differences:

$$
f_{(i)}= \begin{cases}1, & \text { if } \operatorname{sign}\left(\Delta V I_{(i-1, i)}\right)=-\operatorname{sign}\left(\Delta V I_{(i, i+1)}\right) \\ 0, & \text { otherwise }\end{cases}
$$

As specific cases, the peak/valley detector function is set to one at the start point $(i=1)$ and zero at the end point $(i=N)$ of the time series. The signum function $\operatorname{sign}(x)$ equals +1 for $x$ greater than zero, -1 for $x$ less than zero, and zero for $x$ equal to zero. Clearly, the trend direction changes at time points at which the peak/valley detector function equals one (except the time series' start point). These points are called peak and valley points.

For all points of the time series, a second turning point detector function $(g)$, is computed based on the peak/valley detector function and an iterative criterion, explained as follows. In first iteration, for every pair of successive peak or valley points (e.g. $P_{(q)}$ and $P_{(i)}$ or $P_{(i)}$ and $P_{(z)}$ in Fig. 1a), a straight line passing through the two points is computed. Perpendicular distances $(d)$ are computed from all the intermediate non-peak and non-valley points $(f=0)$ to the derived line. On each side of the line (above and below), the intermediate point with maximum perpendicular distance (e.g. $\left.P_{(j)}, P_{(w)}, P_{(l)}\right)$ is selected, and this distance is compared with a user-defined 
distance-threshold $(\varepsilon)$, to be discussed later. If the distance is greater than the threshold (e.g. $d_{j}>\varepsilon$ and $\left.d_{w}>\varepsilon\right)$, the turning point detector function equals one $(g=1)$ at the selected point (i.e. $P_{(j)}$ and $\left.P_{(w)}\right)$, otherwise it is zero $(g=0)$ (e.g. $P_{(l)}$ with $\left.d_{l}<\varepsilon\right)$. At non-selected points, the turning point detector function equals zero as well. So the criterion is fulfilled only at the farthest points above or below the line, provided that its perpendicular distance is above the threshold value.

$g_{(i)}= \begin{cases}1, & \text { if } f_{(i)}=1 \\ 1, & \text { if } f_{(i)}=0 \text { and the criterion is fulfilled. } \\ 0, & \text { if } f_{(i)}=0 \text { and the criterion is not fulfilled. }\end{cases}$

As special cases, the first straight line passes through the time series' start point and the first peak or valley point; similarly the last line passes through the last peak or valley point and the time series' end point. For the second iteration, for every pair of successive points at which $g=1$, a new straight line passing through the two points is computed and the rest proceeds exactly as for the first iteration. The second iteration may add some new points to the set of points at which $g=1$. This same procedure is repeated for further iterations, until no new points with $g=1$ are detected.

Hereafter, time points at which the turning point detector function equals one $(g=1)$ are called turning points: the set of turning points comprises the time series' start point, the peak and valley points and the points at which the criterion is fulfilled (Fig. 1). If $u$ is the number of turning points found for a given time series, then larger values of $u$ indicate more peaks and valleys and/or more points fulfilling the criterion in the trend time series. The turning points' positions (except the time series' start point) indicate times when trend changes occur. Nevertheless, all 
the $u$ turning points are not necessarily associated with substantial changes. As will be discussed later, the user can select changes that have considerable magnitude, after which the significance of the turning points is tested from the fit quality standpoint.

Significant turning points are defined as turning points that significantly reduce the residual sum of squares of a least-square fit to the VI trend time series (explained in next paragraph) and do not result in over-fitting. The number of significant turning points can be determined by minimizing an information criterion. The Bayesian Information Criterion (BIC) (Schwarz, 1978) is a criterion for optimal model selection among a finite set of models. When computing the least-squares fit, adding more turning points reduces the residual sum of squares but also increases the number of model parameters, which may result in over-fitting problem. Both BIC and the Akaike Information Criterion (AIC) (Akaike, 1974) resolve this problem by introducing a penalty term for the number of parameters in the model. We have chosen the BIC because it has been argued that the AIC usually overestimates the number of breaks, while BIC is a suitable selection in many situations (e.g. Zeileis et al., 2002; Zeileis et al., 2003). Given a finite set of estimated models, the preferred or optimal model is the one with the lowest BIC value.

Before using BIC, the trend local change function, designated $h$ and defined in Eq. (5), computes the difference in VI trend between successive turning points, and assigns it to the current turning point: it is zero otherwise for non-turning points. For all points of the time series the function $h$ is defined as follows:

$h_{(i)}= \begin{cases}0, & \text { if } g_{(i)}=0 \\ V I_{(z)}-V I_{(i)}, & \text { if } g_{(i)}=1\end{cases}$ 
where $i$ and $z$ are the indices of the current and next turning points respectively. As two exceptions, we choose $z=N$ for computing $h$ for the last turning point, and $z=2$ for computing $h$ for the first turning point (which is the time series' starting point). All $u$ turning points are then sorted into descending order according to the magnitude of their trend local change (i.e. absolute value of $h$ ). The least-squares fit to the VI trend time series is computed for the entire time series. The fitting is started with no turning point selected from the list to be considered in the fit (i.e. linear fit), and then is repeated for cases where different numbers of the sorted turning points (from just the first, to all $u$ of them) are included in the fit. The BIC is computed for the fit obtained for each case. The significant turning points are specified as the $s(s \leq u)$ points for which the corresponding fit minimizes the BIC.

The least-squares fit to the trend time series is computed using the selected turning points, fitting either one single straight-line (when no turning point is selected) or a composite line (when one or more are selected). In computing the composite line, each selected turning point and the next turning point after it in the trend time series are treated as data points to be shared by the adjoining linear segments. This fitting method leads to a continuous, piecewise trend composed of linear segments. Piecewise linear modelling, as a special case of non-linear regression (Venables and Ripley, 2002), is often used to approximate complex phenomena and extract the basic features of the data (Zeileis et al., 2003; Verbesselt et al., 2010).

The least-squares fitting is explained based on an example. Consider Fig. 1b with 14 data points, where $q=2, j=5, w=6, i=8$, and $z=13$. The $P_{(q)}, P_{(j)}, P_{(w)}, P_{(i)}$, and $P_{(z)}$ turning points are here assumed as data points shared by adjoining segments. The composite line consists of six 
segments (number of the assumed turning points plus one) and is obtained from the following regression model (6).

$$
\begin{aligned}
& V I_{(1)}+\varepsilon_{1}=b \\
& V I_{(2)}+\varepsilon_{2}=a_{1}+b \\
& V I_{(3)}+\varepsilon_{3}=a_{1}+a_{2}+b \\
& V I_{(4)}+\varepsilon_{4}=a_{1}+2 a_{2}+b \\
& V I_{(5)}+\varepsilon_{5}=a_{1}+3 a_{2}+b \\
& V I_{(6)}+\varepsilon_{6}=a_{1}+3 a_{2}+a_{3}+b \\
& V I_{(7)}+\varepsilon_{7}=a_{1}+3 a_{2}+a_{3}+a_{4}+b \\
& V I_{(8)}+\varepsilon_{8}=a_{1}+3 a_{2}+a_{3}+2 a_{4}+b \\
& V I_{(9)}+\varepsilon_{9}=a_{1}+3 a_{2}+a_{3}+2 a_{4}+a_{5}+b \\
& V I_{(10)}+\varepsilon_{10}=a_{1}+3 a_{2}+a_{3}+2 a_{4}+2 a_{5}+b \\
& V I_{(11)}+\varepsilon_{11}=a_{1}+3 a_{2}+a_{3}+2 a_{4}+3 a_{5}+b \\
& V I_{(12)}+\varepsilon_{12}=a_{1}+3 a_{2}+a_{3}+2 a_{4}+4 a_{5}+b \\
& V I_{(13)}+\varepsilon_{13}=a_{1}+3 a_{2}+a_{3}+2 a_{4}+5 a_{5}+b \\
& V I_{(14)}+\varepsilon_{14}=a_{1}+3 a_{2}+a_{3}+2 a_{4}+5 a_{5}+a_{6}+b
\end{aligned}
$$

Rewriting the model in matrix form and utilizing standard methods (Press et al., 1990) gives the values of the coefficients $a_{1}, a_{2}, a_{3}, a_{4}, a_{5}$ and $a_{6}$ (slopes of the six linear segments) and $b$ (model value at the starting data point). Note that the composite line is continuous, but it does not necessarily pass through the turning points considered in fitting. 
The $s$ significant turning points, which minimise the BIC, are called breakpoints. Note that the significant breakpoints, as a subset of the turning points, can be abrupt (i.e. level-shift) or nonabrupt. The breakpoint detection method explained here can therefore be seen as more general than methods in which only abrupt changes are detected, such as standard sequential test-based methods used particularly in econometrics (Bai and Perron, 2003).

The next step allows the user to select either all breakpoints or a subset of them for output fit. The selection depends on the reason for segmentation. If the reason is trend generalisation, there are three options for the selection. If the required trend should reflect only a specified number $m$ $(0 \leq m \leq s)$ of the greatest changes (first option), the first $m$ breakpoints having the largest magnitude of the trend local change are selected. If the trend should contain segments with variations equal to or lower than the user-defined threshold $\beta$ (second option), then the $k$ breakpoints with change magnitudes larger than $\beta$ are selected. The third option is to simplify the trend up to a user-defined level (generalisation-threshold $\delta$ ), compared to the least simplified trend fit derived using all $s$ breakpoints as the base level. In this case, the first $r(r=$ ceil $(s-$ $\delta \times 0.01 \times s))$ breakpoints are selected.

Alternatively, if the reason for segmentation is the detection and characterization of changes (either the $m$ greatest changes, or all changes greater than the magnitude $\beta$ ), then as another field application of the segmentation algorithm, all detected breakpoints $(s)$ will be selected to be considered in the output fit. The user can choose a larger $m$ (or lower $\beta$ or lower $\delta$ ) if their interest is in trend details or short local events, or choose a smaller $m$ (or higher $\beta$ or higher $\delta$ ) if 
their interest is in the main features of the trend, or long-duration processes.

Finally, the least-squares fit to the trend time series (output fit) is computed using the selected breakpoints, fitting either a straight-line (when $m=0$ or $k=0$ or $r=0$ ) or a composite line (when $m$ $\geq 1$ or $k \geq 1$ or $r \geq 1$ ). The outputs of the trend generalisation algorithm are the generalised trend, number of segments, and fit quality measures (root-mean-square-error (RMSE) and maximumabsolute-difference (MAD)). For any detected change, the time of the corresponding breakpoint (which is called the break date) is considered to be the start time, and the time of the next turning point in the trend time series is considered to be the end time. The change duration is the time between the start and end times, and the change value is estimated by subtracting the fitted VI at the start time from the fitted value at the end time. The sign of the change value indicates the change direction, whether the slope is increasing or decreasing. The change type output denotes whether the detected change is abrupt or non-abrupt dependent on whether the corresponding breakpoint is a level-shift point or not, respectively.

An overview of DBEST is shown in Fig. 2, including some practical examples. For instance, in order to generalise a trend time series at maximum level (fit one segment), set $\delta=100 \%$ in the generalisation algorithm: the rest of the analysis is performed as explained above (Fig. 2, left panel, Example 1). As a second example, to generalise the trend into main segments including only one major breakpoint, set $m=1$ (Fig. 2, left panel, Example 2). Thirdly, to generalise into segments containing variations $\leq 0.2$ in NDVI units, set $\beta=0.2$ (Fig. 2, left panel, Example 3). Obviously, setting $\beta=0$ or $m=s$ or $\delta=0$ leads to the least generalised form of the trend (i.e. fit derived using all the $s$ breakpoints). As an example for the change detection algorithm, to detect 
the three major changes set $m=3$ (Fig. 2, right panel, Example 4). Here, all the $s$ breakpoints are used in the fitting, but the outputs are characteristics of only the three changes of interest. This method, which can specify the changes of interest through either the change number or magnitude parameters, preserves the highest fitting quality as well as the actual shape of the change, because the output fit is composed of all significant segments.

As mentioned, the distance-threshold $(\varepsilon)$ is a user-defined parameter, which can be set to a value between zero and maximum absolute difference between data values of the given time series (two for NDVI). However, values larger than the maximum influential value have no effect on the segmentation process. By the maximum influential value we mean the value that if any greater value than it is used for the distance-threshold, no turning points through fulfilling the distance criterion is detected; only peak and valley points plus time series' start point will be detected as turning points (minimum possible $u$ ). The lower the value of $\varepsilon$ relative to the maximum influential value, the larger the number of turning points $(u)$ that will be detected. Setting $\varepsilon=0$ causes all data points to be detected as turning points (maximum possible $u$ ). A default value for $\varepsilon$, however, is estimated in DBEST as follows:

$\varepsilon=3 R^{*}$

where $R^{*}$ is the root mean square error for the fit obtained using the peak and valley points plus the time series' start point (i.e. the distance-threshold is initially set to the maximum absolute difference value). Interpretation of the estimated $\varepsilon$ is that it is a statistical boundary $(\alpha=0.99)$ between outlier and normal residuals of the obtained fit. This means that data points with outlier 
residuals will most likely be detected as turning points through fulfilling the distance criterion, and data points with normal residuals will not be detected. Hence, it is unlikely that noise leads to false detection of turning points because they normally correspond to small residuals.

\subsection{Validation}

DBEST must be tested against remotely sensed satellite imagery in order to investigate its reliability. However, as Verbesselt et al. (2010a) note, the validation of change detection methods is not straightforward. This is frequently due to the unavailability of independent reference sources for a wide range of potential changes during the change interval, and also because the type and number of changes detected are never represented by field validated singledate maps (Kennedy et al., 2007). We therefore simulated NDVI data influenced by different biophysical driving processes and events (both strengthening and disturbing plant greenness, with both abrupt and gradual changes), and different types of plant response, in order to test DBEST robustly under unambiguous and controlled conditions. In addition to using simulated data, validation based on actual satellite data remains necessary because of the challenges existent in perfectly simulating a real environment. Our satellite data were taken from Iraq, where major changes in the NDVI have been observed (Hamdan et al., 2010; Richardson et al., 2005). Fig. 3 illustrates the overall workflow of the validation and evaluation processes.

\subsubsection{Simulation}


We simulated both NDVI trends (without seasonal cycles) and NDVI time series (including seasonal cycles) at two sites with different biomes (forest at site 1 and cropland at site 2 ) for a period of 300 months (corresponding to the real GIMMS data in Iraq) by using artificial processes or events (with typical pre- and post-event life phases) and noise (Table 1). We specified NDVI changes and their durations for each phase, based on observations of corresponding biomes in real conditions.

At site 1, we considered two typical disturbance events in a series of six phases: a stable condition followed by a sudden defoliation (causing a significant level-shift in NDVI), a gradual re-growth phase, a second defoliation, a recovery phase, and finally a return to stable plant greenness in the last phase. At site 2, one plantation and one drought, both with significant impacts on NDVI, were used in respectively second and fourth phases just after stable conditions in first and third phases. Similarly to site 1 , a recovery phase and finally a return to stable plant greenness were considered in respectively fifth and sixth phases (Table 1).

DBEST works for both types of trend (non-seasonal) and VI time series (including seasonal cycles). We therefore simulated both types of data series. For each site, we simulated NDVI trend data by computing a straight-line segment for each phase using an appropriate change and duration to calculate the segment's slope and intercept (Table 1). The initial NDVI levels, as starting points in the first phases, were assumed to be 0.6 (site 1) and 0.15 (site 2). For generating noise - which often originates from geometric and atmospheric errors in satellite data - we used a random number generator with a zero-mean $(\mu=0)$ normal distribution and different values (at least three) for standard deviation ( $\sigma=0.01,0.04,0.07$ NDVI units), corresponding to different 
noise levels (low, medium, strong respectively) (Lhermitte et al., 2011; Roderick et al., 1996). The generated noise for each level was then separately summed with the computed trend at each site. Finally, the derived trends including high frequency variations were smoothed using a locally-weighted regression (loess) smoother (Cleveland and Devlin, 1988) after which likely level-shifts were detected. As recommended for the STL model (Cleveland et al., 1990), we used a first degree polynomial model for the loess smoothing (with a smoothing span of 23 divided by the total number of data points within each part of the time series). For evaluation purposes, the NDVI trends were simulated with 500 iterations for each level of noise at each site.

To generate VI time series with seasonal trends and different types of noise, we simulated seasonal periodic cycles of NDVI at each site, and added them to the earlier simulated noisy trends (before applying the loess smoothing). Finally, we used the separate STL procedure $\left(\theta_{l}=0.1, \theta_{2}=0.2 \mathrm{NDVI}\right.$ units and $\phi=24$ monthly time steps $)$ to detect likely level-shifts and extract trend components. In order to simulate the seasonal component for each biome, we used a symmetric Gaussian function with amplitudes of maximum 0.2 (site 1) and 0.25 (site 2) for healthy seasons. The maximum values were derived by using the STL function for some real forest and cropland GIMMS-NDVI time series from the study area, extracting their seasonal components, and approximating the amplitudes. The Gaussian type function has performed well when used to calculate seasonality parameters by fitting the function to NDVI time series (Jönsson and Eklundh, 2002). The amplitude values used for simulating the disturbed seasons and seasons in the first phase at site 2 were assumed to be lower than the corresponding values for healthy seasons. 


\subsubsection{Spatial application of the major change characterization}

We generated monthly NDVI image series for a period of 25 years (1982-2006) by converting the original twice-monthly GIMMS-NDVI data $(25 \times 12 \times 2=600$ images) to monthly data (300 images) using the maximum-value composite (MVC) method (Holben, 1986), which is similar to the method used in GIMMS. Although the compositing method can reduce cloud and other noise effects, the conversion was solely made because of the easier interpretation of monthly over twice-monthly results, and is not a prerequisite for DBEST. The temporal trend of the monthly NDVI data series was derived using the separate STL decomposition procedure $\left(\theta_{l}=0.1, \theta_{2}=0.2\right.$ NDVI units and $\phi=24$ monthly time steps). We then applied DBEST's change detection algorithm to the trends of the monthly NDVI data series in Iraq in order to detect, estimate, and map major change characteristics (change type, timing and magnitude) during the period 19822006.

\subsection{Evaluation}

To evaluate DBEST's performance under different generalisation schemes, we assigned two values (zero and one) to the number of breakpoints, and two values ( $0.1 \mathrm{NDVI}$ and 0$)$ to the trend local change threshold $(\beta)$, in order to create four different schemes (run settings) respectively called: no-breakpoint, major breakpoint, 0.1 NDVI threshold, and all-inclusive breakpoints. As the fifth scheme, we generalised the trends using different values (from 0 to $100 \%$ ) for the generalisation-threshold $(\delta)$. We then applied DBEST's generalisation algorithm to the simulated trends under these schemes (Fig. 3). For the evaluation, we quantified the 
accuracy of the data fit of each scheme by computing the average RMSE and the MAD between the simulated trend values and the fitted values (on the generalised trend) obtained by DBEST. To do this, the RMSE and MAD values obtained for each individual trend (simulated) were averaged over the 500 iterations of the simulation. In addition, the number of segments in the generalised trend, averaged over the 500 iterations, was computed for each scheme.

Furthermore, we assessed DBEST's performance for detecting and characterizing changes through quantifying the accuracy of the break date, change magnitude estimates and output fits. For this exercise, errors were defined as differences between the estimated (DBEST) and true values (Table 1). First we computed the error of the time and magnitude of the three largest changes detected by DBEST for each individual simulated trend, and calculated the RMSE for the estimates over the 500 iterations. These RMSEs were finally averaged over the three changes. To quantify the data fit accuracy, the average RMSE and MAD for the output fits were computed, in the same way as in the previous paragraph. We also assessed DBEST's performance for determining change type (abrupt or non-abrupt) using the overall accuracy descriptive statistic (e.g. Congalton, 1991). To compute this statistic, the types of the three changes detected by DBEST for each individual trend were compared with the types of the simulated changes. If the change type for all changes was determined correctly we assigned one hundred percent to the statistic for the individual trend, otherwise lower dependent on the number of correct change types. The statistics became zero percent if no change type was determined correctly. We then averaged the statistic over the 500 simulations.

Similarly, all the above mentioned measures for evaluating DBEST performance were computed 
using the simulated NDVI time series. As one exception, the average maximum-absoluteremainder (MAR) was computed instead of MAD, explicitly in order to assess any seasonal variations in fit accuracy.

The average execution time for DBEST was also measured. We ran DBEST (on an ordinary computer with 2.7 GHz Intel Core i7 CPU and 4 Gb RAM) in Matlab (version R2013a).

For comparison purposes, the simulated NDVI time series were used as inputs to both the DBEST and BFAST programs (the most recent version, 1.4.4, is available from http://bfast.rforge.r-project.org/) to detect the largest changes. The average RMSE for the change's estimated time and magnitude (averaged over the 500 iterations) and the overall accuracy for type of the detected change were similarly computed for each method (Fig. 3). In BFAST, the harmonic season type option was selected for the simulated NDVI time series. The minimal segment size parameter $(h)$ was left as default, and the maximum iteration parameter (max.iter) was set to three.

All the evaluation and method comparison computations (summarized in Fig. 3) were repeated for each noise level, in order to assess the models' robustness. Finally, we used DBEST to detect and map major changes in the Iraq data, as a practical application of the method.

\section{Results and discussion}




\subsection{Validation and evaluation using the simulated data}

\subsubsection{Trend generalisation}

DBEST's trend generalisation performance was tested using the five schemes described in section 3.4. Fig. 4 shows results for one sample of the simulated trends for each site. The nobreakpoint ( $\mathrm{m}=0$ or $\delta=100 \%$ ) scheme produced the most generalised fits (straight-lines) (Fig. 4a, f). The major events were successfully captured using the major breakpoint scheme (Fig. 4b, g), which represented the data by three segments, of which the central ones represented the major event (the sudden defoliation at site 1, and planting at site 2). By looking for magnitude changes of 0.1 NDVI (Fig. 4c, h), the number of detected breakpoints increased to $k=3$, meaning that more segments (6) were generated. The all-inclusive breakpoints scheme generated the highest number of segments (14 segments for site 1 and 15 for site 2), and the least generalised fits $(\delta=0)$, while preserving details about minor events that carry significant information as confirmed by BIC (Fig. 4d, i). However, these minor events should be carefully interpreted as they may be artefacts produced by trend analysis in the presence of underlying environmental trends other than VI, as stated by Wessels et al. (2012).

As expected, the no-breakpoint scheme led to the lowest fit accuracies for all noise levels (average RMSE 0.10-0.13 NDVI, and average MAD 0.16-0.23 NDVI). The major breakpoint scheme was much more accurate (by about $76 \%$ for the average RMSE and $54 \%$ for the average MAD). Segmentation using the threshold scheme (0.1 NDVI) ranked second, closely after the all-inclusive breakpoints scheme that always ranked first (average RMSE $<0.01$ and 
MAD $<0.03$ NDVI). In all schemes, higher noise levels had a slightly negative impact on the fit accuracy (average RMSE and average MAD).

For all noise levels at both sites, the number of segments in the generalised trends decreased almost linearly when the generalisation-threshold $(\delta)$ increased from zero to one hundred percent (results are not shown). However, the average number of segments was smaller for lower noise levels. The results demonstrated DBEST's efficiency in generalising trends, which carries the associated benefit of reducing the cost of further computations based on the generalised trend.

The results of evaluating different generalisation schemes using the simulated NDVI time series are not shown, since they are broadly similar to the results obtained using the simulated NDVI trends.

\subsubsection{Detecting and characterizing trend change}

Fig. 5 illustrates one sample of the simulated NDVI time series for each site, with the decomposed components (trend, seasonal and remainder), and three major changes detected by DBEST. Note that the simulated trends without noise (in green) which represent the plant life phases were used as basis for simulating the NDVI time series (Fig. 5a, e). The seasonal components contained seasonal growing cycles with varying amplitudes for healthy and disturbed seasons (Fig. 5c, g). The remainders (unexplained variance) were, in general, small (Fig. 5d, h). Since DBEST's main purpose is currently for detecting changes in the trend component, we keep the focus on the trend results. 
We chose to detect three breakpoints at each site. As intended, DBEST captured the processes and/or events that caused the greatest changes to the NDVI trend at both sites (the two defoliations and one post disturbance recovery process at site 1; and the plantation, drought, and post drought recovery at site 2); and, approximated them with segments sloped properly (either abrupt as the first sudden defoliation at site 1 or slower as the other changes) and matched adequately in shape to the trend (Fig. 5b, f).

Break dates were determined by DBEST with an average RMSE range of 2.7-3.9 time steps at site 1 (Fig. 6a), and 2.5-4.9 time steps at site 2 (the less accurate estimates correspond to higher noise levels) (Fig. 6b). Higher noise levels had a slightly more negative impact on the average RMSE of the time at site 2 than site 1 . The more robust results obtained for site 1 might be because of the larger underlying changes, and hence higher signal to noise ratios, compared to site 2 (Table 1). Another reason may be due to the type of the first change (abrupt level-shift) at site 1; DBEST showed more accurate results for level-shift changes (discussed later on in section 4.1.3.). The change magnitude accuracies were $~ 0.04-0.05$ NDVI units at both sites (Fig. 6a, b). The average RMSE for obtained fits was 0.02-0.06 (NDVI units) at both sites, and the average MAR was 0.09-0.18 (NDVI units) at both sites. The fit accuracy measures (average RMSE and average MAR) decreased slightly as the noise level increased (Fig. 6a, b).

The overall accuracy for the change type determination was in the range $95-100 \%$ at site 1 and 87-100\% at site 2, with lower accuracies at higher noise levels. The results of evaluating DBEST for detecting and characterizing the three changes using the simulated trends are not shown, since they are generally similar to the results obtained using the simulated NDVI time series. 


\subsubsection{Comparison with BFAST}

Both the DBEST and BFAST methods showed accurate estimates (average RMSE $\leq 2$ time step) for the timing of major changes (i.e. sudden defoliation) detected at site 1. BFAST showed more accurate estimates by about 1-1.7 time steps for the $\sigma=0.04-0.07$ noise levels, respectively, but both methods estimated the change time with almost no error at the 0.01 noise level (Fig. 7a). At site 2 with the planting as the major change, both methods showed less accurate estimates than site 1 (average RMSEs of 1-3.5 time steps for DBEST and about 7 time steps for BFAST). However, DBEST showed improvements by about 3.5-6 time steps compared to BFAST (lower noise levels giving the bigger improvement).

The major change, at both sites, was estimated with an average RMSE of 0.02-0.07 NDVI units for DBEST and 0.03-0.05 NDVI units for BFAST (higher accuracy for lower noise levels). The change was estimated by BFAST by about 1-3\% NDVI units more accurate than DBEST, except at site 1 where DBEST showed $\sim 1 \%$ NDVI improvement for the 0.01 noise level (Fig. 7b). Again, both methods showed more accurate estimates for the abrupt change at site 1 than the non-abrupt change at site 2 .

BFAST correctly approximated the sudden defoliation at site 1 with a sudden break at all three noise levels (one hundred percent overall accuracy). DBEST did similarly for the lowest noise (0.01), but by about $8 \%$ and $13 \%$ weaker overall accuracy for the 0.04 and 0.07 noise levels (Fig. 7c). BFAST approximated the planting change at site 2 with a sudden break too (like site 1) 
lasting for one time-step while the actual duration was much longer (12 time steps), whereas DBEST showed a significant improvement (63-100\%) by correctly determining the non-abrupt change type. In the real world some natural processes may have long-term influences on the vegetation trend, and cause monotonic changes in the NDVI trend. DBEST approximates such changes with segments possessing more realistic slopes, i.e. rates of change (as seen in Fig. 5b, f). An important point that should not be forgotten here is that the methods may have no identical definition of breakpoint for detection; breakpoints normally imply discontinuity or break (abrupt change) in BFAST (Verbesselt et al., 2010a) whereas a breakpoint can include both abrupt and non-abrupt changes in DBEST.

The main advantages of BFAST, however, are that it computes a confidence interval for the change time estimate and detects changes in the seasonal component, whereas DBEST is not yet developed for these tasks. On other hand, DBEST detects both abrupt and non-abrupt changes with comparable accuracy for abrupt changes and improved accuracy for non-abrupt changes, and it has a generalisation algorithm for flexible extraction of information at any scale, from details to main trend features.

\subsection{User-defined thresholds and parameters}

The level-shift thresholds $\left(\theta_{1}\right.$ and $\left.\theta_{2}\right)$ and the duration-threshold $(\phi)$, used for the detection of level-shift changes, are set based on the user's criteria for detecting abrupt changes of interest in the studied variable. We considered a change in NDVI as a significant level-shift when it was greater than 0.1 NDVI units $\left(\theta_{l}=0.1\right)$, when it shifted the level (mean) of NDVI by more than 0.2 
$\left(\theta_{2}=0.2\right)$, and finally when the shift was valid for at least two years ( $\phi=24$ monthly time steps).

The user can change these threshold parameters to suit their own data set, but it is recommended that $\theta_{1 \leq} \theta_{2}$. Setting larger level-shift thresholds $\left(\theta_{1}\right.$ and $\left.\theta_{2}\right)$ and a longer duration-threshold $(\phi)$ is safer than smaller level-shift thresholds and shorter duration-threshold setting, because the latter may lead to false detection of NDVI seasonal variations.

To check the effect on DBEST of the distance-threshold parameter $(\varepsilon)$, we computed the accuracies (in terms of average RMSE over the 500 iterations) of the estimated times and magnitudes of the three major changes in the simulated trend time series at the two sites using a noise setting of 0.04 , using the values $\varepsilon=0-0.2$ NDVI units (Fig. 8). As seen, the accuracies improve (RMSEs decrease) as the distance-threshold increases from 0.01 to around $0.02-0.03$ for time and 0.06-0.07 for magnitude, but decrease (RMSEs increase except for the magnitude at site 1) before levelling off at around 0.1 NDVI units (this should be the maximum influential value of distance-threshold). For $\varepsilon$ values up to 0.1 , the time estimates showed higher sensitivity to higher values of $\varepsilon$ whereas the magnitude estimates showed higher sensitivity to lower $\varepsilon$ values. We then recomputed the accuracies using the $\varepsilon$ values derived by Eq. (7) in DBEST. The derived value for the distance-threshold, averaged over the 500 simulations, was about 0.06 at site 1 and 0.04 at site 2 (markers in red in Fig. 8); they were close to the $\varepsilon$ values for which the lowest RMSEs were obtained. The computed accuracies (using $\varepsilon$ values derived by Eq. (7)) were also comparable with the highest accuracies achieved. Similar results were obtained for noise levels of $\sigma=0.01$ and $\sigma=0.07$. These demonstrate that Eq. (7) works satisfactorily. The user can choose to keep the default value of the distance-threshold derived by Eq. (7) in DBEST, or change it to a 
value between zero and maximum absolute difference between data values of the given time series.

The generalisation-threshold $(\delta$ ) parameter can be freely set by the user (between 0 and 100\%) and no specific default is set in DBEST for this parameter. The same goes for the changemagnitude-threshold $(\beta)$ and the number of change of interest $(m)$ parameters. The only exception is that the $m$ parameter can be no larger than the total number of significant breakpoints $(s)$ determined by BIC.

\subsection{Spatial application of the major change characterization}

When detecting changes in Iraq, the major change detected in the NDVI trend was of the nonabrupt change type over the whole country. The exception was in small regions in the southeast close to the border with Iran, which showed abrupt (i.e. level-shift) positive changes (Fig. 9a). The non-abrupt changes were fairly small $(\leq 0.1$ NDVI units) over most parts of the area (89\% of unmasked area) (Fig. 9b), either positive (48\%) mostly in west or negative (41\%) scattered elsewhere, but mainly in east and northeast. Some scattered small areas with small positive changes were also found within the rest of the area that showed negative changes. Bigger positive changes (between 0.1 and 0.2 in NDVI units) were detected in north Iraq (5\%). Surrounding them, there were also detected areas (5\%) with considerable negative changes (between -0.1 and -0.3 in NDVI units) (Fig. 9a). 
The small changes (<0.1 NDVI) started mainly in 1995-1998 and lasted for a 2-24 month time period (Fig. 9c, d). The northern greening started in 1984-1988 and lasted for 12-36 months in most parts. The browning seen in the north and northeast, surrounding the greening, started in 1987 and lasted mainly for a 2-12 month period, or a few months longer. This gradual browning could reflect the impact of huge emigration in the 1990s (Sirkeci, 2005). The strong greening in NDVI (>0.2) in the southeast close to the border with Iran $(\sim 1 \%)$, identified mainly as abrupt change (e.g. Fig. 9e) with a one-month duration or some few non-abrupt changes with longer durations (2-12 months) in neighbouring pixels, started in 2004-2005.

Our findings are generally in agreement with Hamdan et al., (2010) and Richardson et al., (2005); they reported a similar vegetation response in the south-eastern wetlands due to a replenishment and releasing of the Tigris and Euphrates River waters, and another extensive reflooding in March 2004. The uncontrolled replenishment was implemented by local residents following the fall of Iraq's government in spring 2003, after a long period of deliberate destruction and wetland reduction by Iraq's government during 1990.

Following this confirmation, the regions with large NDVI changes may be targeted for further investigation, using ground data and finer resolution imagery. This is because there is always a possibility that some of the observed changes are due to factors other than change in vegetation. One possible source of error is that the GIMMS data set comprises data from six NOAA satellites: orbital drift of the satellites can distort the calculated NDVI, leading to false change detection. 


\subsection{Pros and cons}

DBEST is independent of the sensors that provide its input data. It can be applied to image series of any vegetation index, on a pixel-by-pixel basis, regardless of spatial and temporal resolution. DBEST's main outcomes (i.e. fitted segments) are useful for estimating the characteristics (time and magnitude) of abrupt and long-duration changes, for comparing long term vegetation trends for different locations, and for comparing NDVI trends observed by different sensors. DBEST also has the potential to be employed more generally in multiple breakpoint detection, an active research field in statistics and econometrics (Reeves et al., 2007).

However, there are two issues that require attention when applying DBEST. First, if input data to DBEST are deseasonalized time series, they should not include high frequency variation (local roughness): this criterion should be fulfilled because the trend, by definition, is a slowly changing, smooth component of a time series. Otherwise, it is recommended to remove the variations using standard smoothing filters. Second, DBEST works on a pixel-by-pixel basis and considers each pixel's time series data as an isolated entity in the change detection procedure; the spatial behaviour of adjacent areas is not used to improve robustness of detection. The use of neighbouring pixels for robust trend estimation of remotely sensed time series data is demonstrated by Bolin et al. (2009).

DBEST has simple mathematical principles and as a software product runs correspondingly quickly (0.06-0.17 sec average execution time to detect change using the simulated trend and 
NDVI time series, respectively). The slower speed in the latter case is mainly due to the time it takes for the STL to run.

The DBEST user can control the trend generalisation and change detection processes through a number of thresholds and parameters, including the generalisation percentage, the number of required changes and the change threshold parameters.

\subsection{Future work}

Further development of DBEST will include testing it using remotely sensed satellite imagery for different types of changes in a wide range of ecosystems. It would be interesting, from the standpoint of ecosystem management or warning system development, to investigate DBEST's sensitivity to different trend types (e.g. monotonic, interrupted, reversal trends, or trends initiated from a steady-state equilibrium), and change magnitudes. In addition, validation under noisier conditions (caused by clouds or snow), using images with finer temporal and spatial resolution, still remains necessary. Moreover, the current version of DBEST can only analyse regularly spaced data, in the temporal sense. Development and testing DBEST using irregularly spaced data series (including time series with missing data), as well as the detection of changes in the seasonal component of a time series, are next in line as future works.

It is our intention to integrate DBEST with TIMESAT (Jönsson and Eklundh, 2004) to enable non-linear trend detection to be applied to remotely sensed seasonality parameters. 


\section{Conclusions}

Our recently developed time series algorithm DBEST can properly simplify the temporal trend of a vegetation index time series into a flexible number of straight-line segments. The user can control the generalisation process in DBEST by setting the generalisation-threshold. This capability allows the user to capture a wide range of features, from fine details through to main features of the trend, over large areas. DBEST also detects trend changes, determines their type (abrupt or non-abrupt), and estimates their timing, magnitude, number, and direction. The user can set the number or magnitude of major changes for detection.

The validation and evaluation results demonstrated DBEST's efficiency in terms of providing results which were accurate, robust and cost effective. We used DEBST to detect major changes in NDVI trend over Iraq during 1982-2006; it was small (<0.1 NDVI) over large areas and in both forms of gradual greening (48\% of the land area, mostly in the western regions) and browning ( $41 \%$ of the land area). Northern and eastern Iraq showed larger changes as both

greening (0.1-0.2 NDVI) and browning (-0.1- -0.3 NDVI) (5\% each). Abrupt changes ( $>0.2$ NDVI) leading to significant shifts in the level of NDVI were detected in relatively small areas $(\sim 1 \%)$ in southeast.

DBEST can be applied to the time series of different remotely sensed vegetation indices for detecting and mapping both short and long duration events at different spatial and temporal scales. DBEST also has the potential to be applied in other disciplines dealing with multiple 
trend change detection, such as hydrology and climatology. The MATLAB code developed in this paper is available by contacting the authors.

\section{Acknowledgments}

The authors are very grateful for the constructive feedback from two anonymous reviewers that helped improve the quality of the article. The authors are also grateful to Dr. Jan Verbesselt for clarifications regarding the functionality of BFAST. They also thank Dr. Ford Cropley for proofreading the final version. The authors appreciate the NASA GIMMS group for providing the NDVI data at no cost. This work was supported by European Union funding program, Erasmus Mundus “External Cooperation Window” (EMECW lot8). Partial support was also provided by LUCID (www.lucid.lu.se), a Linnaeus centre of excellence at Lund University.

\section{References}

Akaike, H. (1974). A new look at the statistical model identification. IEEE Transactions on Automatic Control, 19, 716-723.

Bai, J., \& Perron, P. (2003). Computation and analysis of multiple structural change models. Journal of Applied Econometrics, 18(1), 1-22. 
Bolin, D., Lindström, J., Eklundh, L. \& Lindgren, F. (2009). Fast estimation of spatially dependent temporal vegetation trends using Gaussian Markov Random Fields. Computational Statistics and Data Analysis, 53, 2885-2896.

Carlson, T.N., \& Ripley, D.A. (1997). On the relation between NDVI, fractional vegetation cover, and leaf area index. Remote Sensing of Environment, 62, 241-252.

Cleveland, R.B., Cleveland, W.S., McRae, J.E., \& Terpenning, I. (1990). STL: A seasonal-trend decomposition procedure based on loess. Journal of official statistics, 6, 3-73.

Cleveland, W.S., \& Devlin, S.J. (1988). Locally weighted regression - an approach to regression-analysis by local fitting. Journal of the American Statistical Association, 83, 596-610.

Congalton, R.G. (1991). A review of assessing the accuracy of classifications of remotely sensed data. Remote Sensing of Environment, 37, 35-46

Cohen, W.B., Yang, Z., \& Kennedy, R. (2010). Detecting trends in forest disturbance and recovery using yearly Landsat time series: 2. TimeSync - Tools for calibration and validation. Remote Sensing of Environment, 114, 2911-2924.

de Jong, R., Verbesselt, J., Schaepman, M.E., \& de Bruin, S. (2012). Trend changes in global greening and browning: contribution of short-term trends to longer-term change. Global Change Biology, 18, 642-655. 
de Jong, R., Verbesselt, J., Zeileis, A., \& Schaepman, M. (2013). Shifts in Global Vegetation Activity Trends. Remote Sensing, 5(3), 1117-1133, (doi:10.3390/rs5031117).

Draxler, R.R., Gillette, D.A., Kirkpatrick, J.S., \& Heller, J. (2001). Estimating PM10 air concentrations from dust storms in Iraq, Kuwait and Saudi Arabia. Atmospheric Environment, $35,4315-4330$.

Eklundh, L., \& Olsson, L. (2003). Vegetation index trends for the African Sahel 1982-1999. Geophysical Research Letters, 30(8), 1430, (doi:10.1029/2002GL016772).

Fan, L., Gao, Y., Brueck, H., \& Bernhofer, C. (2009). Investigating the relationship between NDVI and LAI in semi-arid grassland in Inner Mongolia using in-situ measurements. Theoretical and Applied Climatology, 95, 151-156.

Fensholt, R., Langanke, T., Rasmussen, K., Reenberg, A., Prince, S.D., Tucker, C., Scholes, R.J., Le, Q.B., Bondeau, A., Eastman, R., Epstein, H., Gaughan, A.E., Hellden, U., Mbow, C., Olsson, L., Paruelo, J., Schweitzer, C., Seaquist, J., \& Wessels, K. (2012). Greenness in semi-arid areas across the globe 1981-2007 — an Earth Observing Satellite based analysis of trends and drivers. Remote Sensing of Environment, 121, 144-158.

Fensholt, R., Rasmussen, K., Nielsen, T.T., \& Mbow, C. (2009). Evaluation of earth observation based long term vegetation trends - Intercomparing NDVI time series trend analysis consistency 
of Sahel from AVHRR GIMMS, Terra MODIS and SPOT VGT data. Remote Sensing of Environment, 113, 1886-1898.

Fuller, D.O. (1998). Trends in NDVI time series and their relation to rangeland and crop production in Senegal, 1987-1993. International Journal of Remote Sensing, 19, 2013-2018.

Hamdan, M., Asada, T., Hassan, F., Warner, B., Douabul, A., Al-Hilli, M., \& Alwan, A. (2010). Vegetation Response to Re-flooding in the Mesopotamian Wetlands, Southern Iraq. Wetlands, $30,177-188$.

Holben, B.N. (1986). Characteristics of maximum-value composite images from temporal AVHRR data. International Journal of Remote Sensing, 7, 1417-1434.

Heumann, B.W., Seaquist, J.W., Eklundh, L., \& Jonsson, P. (2007). AVHRR derived phenological change in the Sahel and Soudan, Africa, 1982-2005. Remote Sensing of Environment, 108, 385-392.

Higgins, P.A.T., Mastrandrea, M.D., \& Schneider, S.H. (2002). Dynamics of climate and ecosystem coupling: abrupt changes and multiple equilibria. Philosophical Transactions of the Royal Society of London Series B-Biological Sciences, 357, 647-655. 
Jacquin, A., Sheeren, D., \& Lacombe, J.P. (2010). Vegetation cover degradation assessment in Madagascar savanna based on trend analysis of MODIS NDVI time series. International Journal of Applied Earth Observation and Geoinformation, 12S, S3-S10.

Jamali, S., Seaquist, J., Eklundh, L., \& Ardö, J. (2014). Automated mapping of vegetation trends with polynomials using NDVI imagery over the Sahel. Remote Sensing of Environment, 141, 7989.

Jönsson, P., \& Eklundh, L. (2002). Seasonality extraction by function fitting to time-series of satellite sensor data. Geoscience and Remote Sensing, IEEE Transactions on, 40, 1824-1832.

Jönsson, P., \& Eklundh, L. (2004). TIMESAT - a program for analyzing time-series of satellite sensor data. Computers \& Geosciences, 30, 833-845.

Kennedy, R.E., Cohen, W.B., \& Schroeder, T.A. (2007). Trajectory-based change detection for automated characterization of forest disturbance dynamics. Remote Sensing of Environment, 110, $370-386$.

Kennedy, R.E., Yang, Z., \& Cohen, W.B. (2010). Detecting trends in forest disturbance and recovery using yearly Landsat time series: 1. LandTrendr - Temporal segmentation algorithms. Remote Sensing of Environment, 114, 2897-2910. 
Keogh, E., Chu, S., Hart, D., \& Pazzani, M. (2004). Segmenting time series: A survey and novel approach. Data mining in time series databases, 57, 1-22.

Lambin, E.F., \& Ehrlich, D. (1997). Land-cover changes in sub-Saharan Africa (1982-1991): Application of a change index based on remotely sensed surface temperature and vegetation indices at a continental scale. Remote Sensing of Environment, 61, 181-200.

Lambin, E.F., Geist, H.J., \& Lepers, E. (2003). Dynamics of land-use and land-cover change in tropical regions. Annual Review of Environment and Resources, 28, 205-241.

Lhermitte, S., Verbesselt, J., Verstraeten, W.W., \& Coppin, P. (2011). A comparison of time series similarity measures for classification and change detection of ecosystem dynamics. Remote Sensing of Environment, 115, 3129-3152.

Nemani, R.R., Keeling, C.D., Hashimoto, H., Jolly, W.M., Piper, S.C., Tucker, C.J., Myneni, R.B., \& Running, S.W. (2003). Climate-driven increases in global terrestrial net primary production from 1982 to 1999 . Science, 300, 1560-1563.

Peng, J., Liu, Z., Liu, Y., Wu, J., \& Han, Y. (2012). Trend analysis of vegetation dynamics in Qinghai-Tibet Plateau using Hurst Exponent. Ecological Indicators, 14, 28-39.

Prentice, I.C., Heimann, M., \& Sitch, S. (2000). The carbon balance of the terrestrial biosphere: Ecosystem models and atmospheric observations. Ecological Applications, 10, 1553-1573. 
Press, W.H., Flannery, B.P., Teukolsky, S.A., \& Vetterling, W.T. (1990). Numerical recipes. Cambridge University Press.

Reeves, J., Chen, J., Wang, X.L., Lund, R., \& Lu, Q. (2007). A review and comparison of changepoint detection techniques for climate data. Journal of Applied Meteorology and Climatology, 46, 900-915.

Richardson, C.J., Reiss, P., Hussain, N.A., Alwash, A.J., \& Pool, D.J. (2005). The restoration potential of the Mesopotamian marshes of Iraq. Science, 307, 1307-1311.

Roderick, M., Smith, R., \& Cridland, S. (1996). The precision of the NDVI derived from AVHRR observations. Remote Sensing of Environment, 56, 57-65.

Rouse, J.W., Hass, R.H., Schell, J.A., \& Deering, D.W. (1973). Monitoring vegetation systems in the Great Plains with ERTS. Third ERTS Symposium, NASA SP-351 I, 309-317.

Schnepf, R. (2004). Iraq Agriculture and Food Supply: Background and Issues. In, CRS Report for Congress, Order Code RL32093, Congressional Research Service, The Library of Congress Congressional Research Service, The Library of Congress.

Schwarz, Gideon E. (1978). Estimating the dimension of a model. Annals of Statistics 6 (2): $461-464$. 
Sirkeci, I. (2005). War in Iraq: Environment of insecurity and international migration. International Migration, 43, 197-214.

Sitch, S., Smith, B., Prentice, I.C., Arneth, A., Bondeau, A., Cramer, W., Kaplan, J.O., Levis, S., Lucht, W., Sykes, M.T., Thonicke, K., \& Venevsky, S. (2003). Evaluation of ecosystem dynamics, plant geography and terrestrial carbon cycling in the LPJ dynamic global vegetation model. Global Change Biology, 9, 161-185.

Tucker, C.J., Pinzon, J.E., \& Brown, M.E. (2004). Global Inventory Modeling and Mapping Studies. In: Global Land Cover Facility, University of Maryland, College Park, Maryland .

Tucker, C.J., Pinzon, J.E., Brown, M.E., Slayback, D.A., Pak, E.W., Mahoney, R., Vermote, E.F., \& El Saleous, N. (2005). An extended AVHRR 8-km NDVI dataset compatible with MODIS and SPOT vegetation NDVI data. International Journal of Remote Sensing, 26, 44854498.

UNEP (2001). The Mesopotamian Marshlands: Demise of an Ecosystem. In: Division of Early Warning and Assessment, United Nations Environment Programme, Nairobi, Kenya.

Verbesselt, J., Hyndman, R., Newnham, G., \& Culvenor, D. (2010a). Detecting trend and seasonal changes in satellite image time series. Remote Sensing of Environment, 114, 106-115. 
Verbesselt, J., Hyndman, R., Zeileis, A., \& Culvenor, D. (2010b). Phenological change detection while accounting for abrupt and gradual trends in satellite image time series. Remote Sensing of Environment, 114, 2970-2980.

Verbesselt, J., Robinson, A., Stone, C., \& Culvenor, D. (2009). Forecasting tree mortality using change metrics derived from MODIS satellite data. Forest Ecology and Management, 258, 11661173.

Venables, W. N., \& Ripley, B. D. (2002). Modern applied statistics with S (pp. 156-163)., 4th Edition. Springer-Verlag.

Wessels, K.J., van den Bergh, F., \& Scholes, R.J. (2012). Limits to detectability of land degradation by trend analysis of vegetation index data. Remote Sensing of Environment, 125, 1022

Zeileis, A., Leisch, F., Hornik, K., \& Kleiber, C. (2002). strucchange: An R package for testing for structural change in linear regression models. Journal of Statistical Software, 7(2), 1-38.

Zeileis, A., Kleiber, C., Kramer, W., \& Hornik, K. (2003). Testing and dating of structural changes in practice. Computational Statistics \& Data Analysis, 44, 109-123. 


\section{Tables}

Table 1. Magnitude and duration (year) of changes used for simulating trends of monthly NDVI at site 1 (forest) and site 2 (cropland).

\begin{tabular}{lllllll}
\hline & $\begin{array}{l}\text { 1st } \\
\text { phase }\end{array}$ & 2nd phase & 3rd phase & 4th phase & 5th phase & $\begin{array}{l}\text { 6th } \\
\text { phase }\end{array}$ \\
\hline Site 1 & Stability & $\begin{array}{l}\text { Sudden } \\
\text { defoliation }\end{array}$ & $\begin{array}{l}\text { Gradual } \\
\text { regrowth }\end{array}$ & Defoliation & Recovery & Stability \\
\hline Change/Duration & $0 / 5$ & $-0.4 /(1 / 12)$ & $0.15 / 9$ & $-0.2 / 2$ & $0.3 / 2$ & $0 / 6$ \\
\hline Site 2 & Stability & Planting & Stability & Drought & Recovery & Stability \\
\hline Change/Duration & $0 / 5$ & $0.3 / 1$ & $0 / 13$ & $-0.2 / 2$ & $0.15 / 2$ & $0 / 2$ \\
\hline
\end{tabular}




\section{Figures}


Fig. 1. A schematic representation of part of a given VI trend time series, in which $P_{(i)}$ denotes the vegetation index value at time-point $i$. (a) - consecutive original points (solid in black), straight-lines (dashed in black) passing through pairs of consecutive peak and valley points at which $f=1$ (e.g. $P_{(q)}, P_{(i)}$ and $P_{(z)}$ ), perpendicular distances from other points $(f=0)$ to the straight-lines (e.g. $\left.d_{(j)}, d_{(w)}, d_{(l)}\right)$, and the turning points (rings in red). (It is assumed that $d_{j}>\varepsilon, d_{w}>\varepsilon$, and $d_{l}<\varepsilon$ ). (b) - the model trend (segments in red) obtained by DBEST when the change detection algorithm is used, which detects $P_{(j)}$ as the major change point. All the turning points (except the time series' start point) are shared by adjoining segments. 


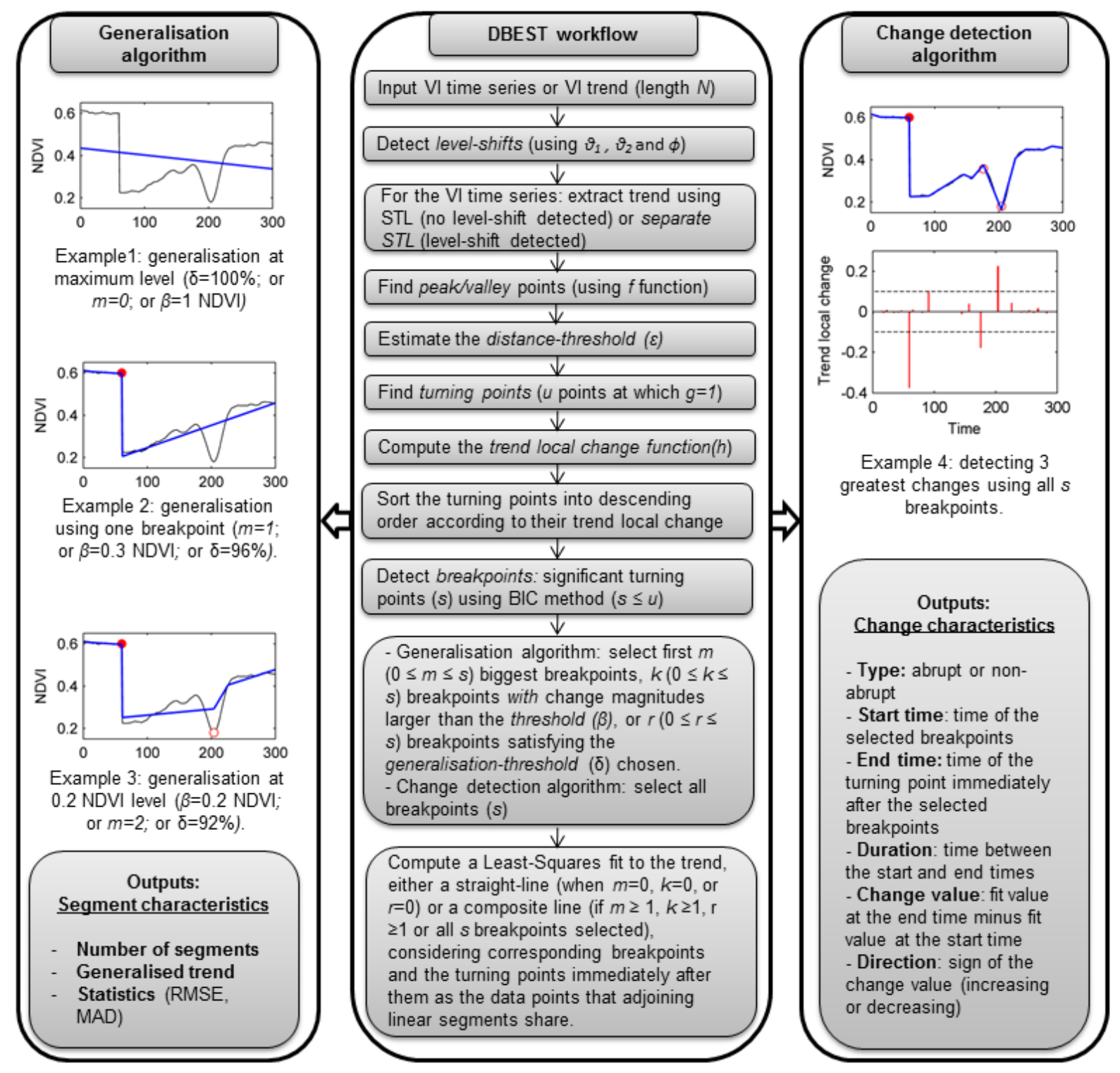

Fig. 2. Flowchart of DBEST for the two main applications of generalising trends (left panel), and detecting and characterizing changes (right panel) in VI time series. 


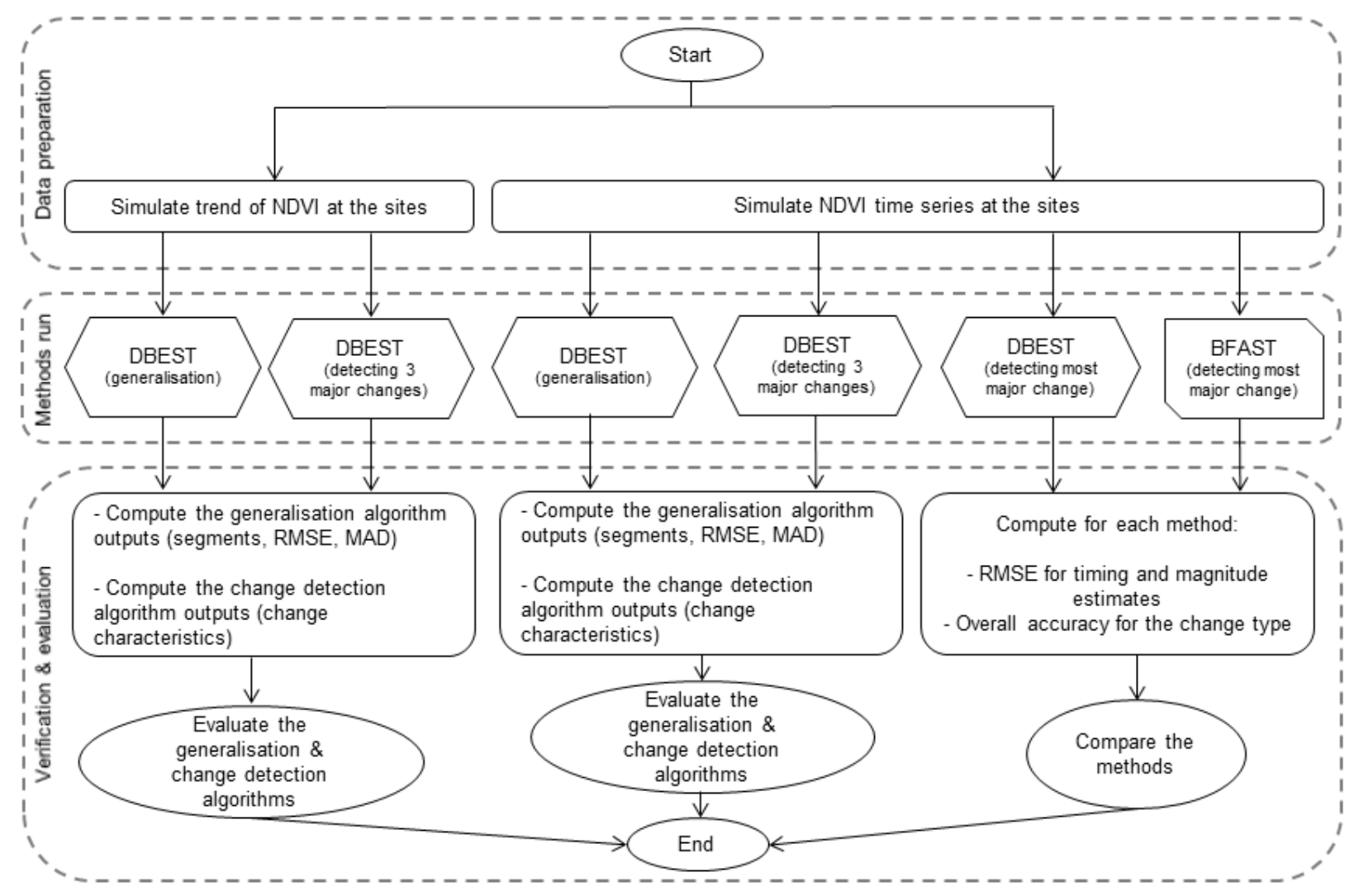

Fig. 3. Validation and evaluation workflow for DBEST. All computations were repeated for each level of noise $(\sigma=0.01,0.04,0.07)$. RMSE $=$ Root Mean Squares Error, MAD $=$ Maximum Absolute Difference. 
Sub-figures (a, b, c, d, e), on left-hand column
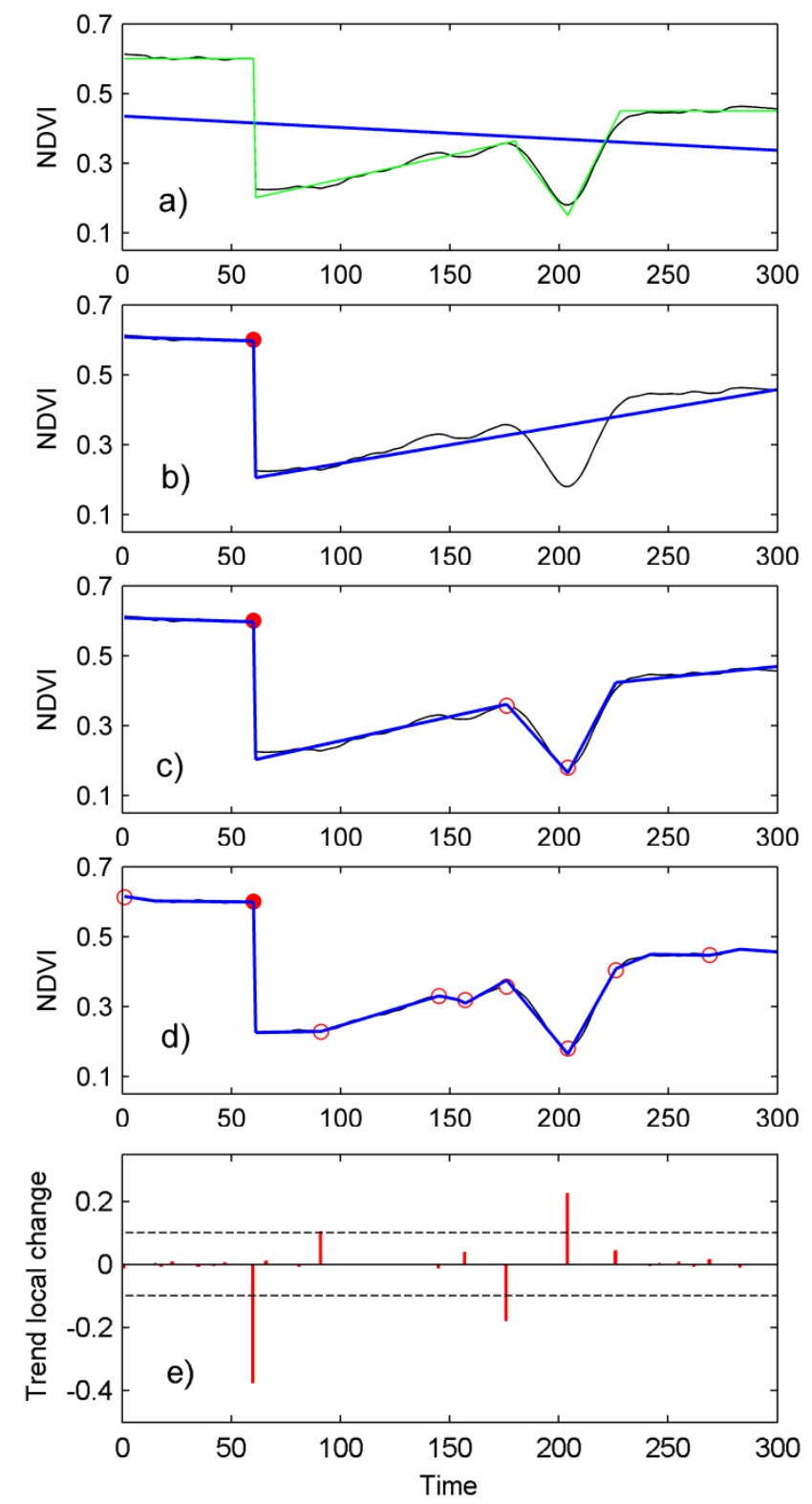
Sub-figures (f, g, h, i, j), on right-hand column

Fig. 4. Left-hand column shows results of DBEST's trend generalisation algorithm for site 1, right-hand column for site 2. The plots show simulated trends of NDVI at $\sigma=0$ (in green) and $\sigma=0.04$ noise levels, and the model fits (in blue) obtained by applying DBEST. The DBEST generalisation schemes used were: (a, f) no-breakpoint, (b, g) major breakpoint, $(\mathrm{c}, \mathrm{h})$ change magnitude-threshold $\beta=0.1 \mathrm{NDVI}$, and $(\mathrm{d}, \mathrm{i})$ all-inclusive breakpoints schemes at site 1 and site 2. Bar charts of the trend local change (i.e. $h$ function) with 0.1 NDVI boundaries (dashed lines) are also shown $(e, j)$. Every turning point has a trend local change value on the bar-charts, so the higher the absolute trend change value, the higher the probability that the point is a major breakpoint. Rings in red denote detected breakpoints in each scheme; solid rings represent level-shifts (abrupt breakpoint), and empty rings represent nonabrupt breakpoints. The simulated NDVI trend, shown for each site, is one of the 500 iterations of the simulation using $\sigma=0.04$ noise level. The trend local change values are in NDVI units. 
Sub-figures (a, b, c, d), on left-hand column
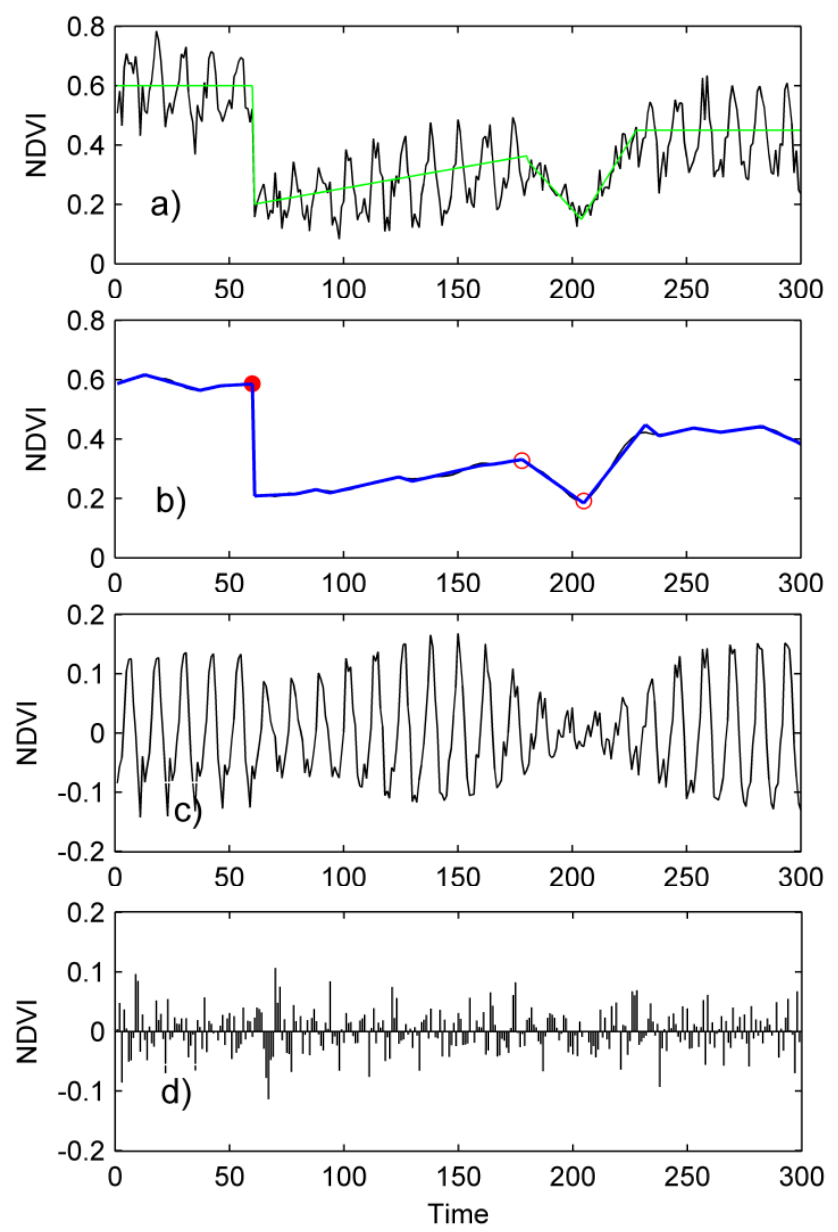
Sub-figures (e, f, g, h), on right-hand column
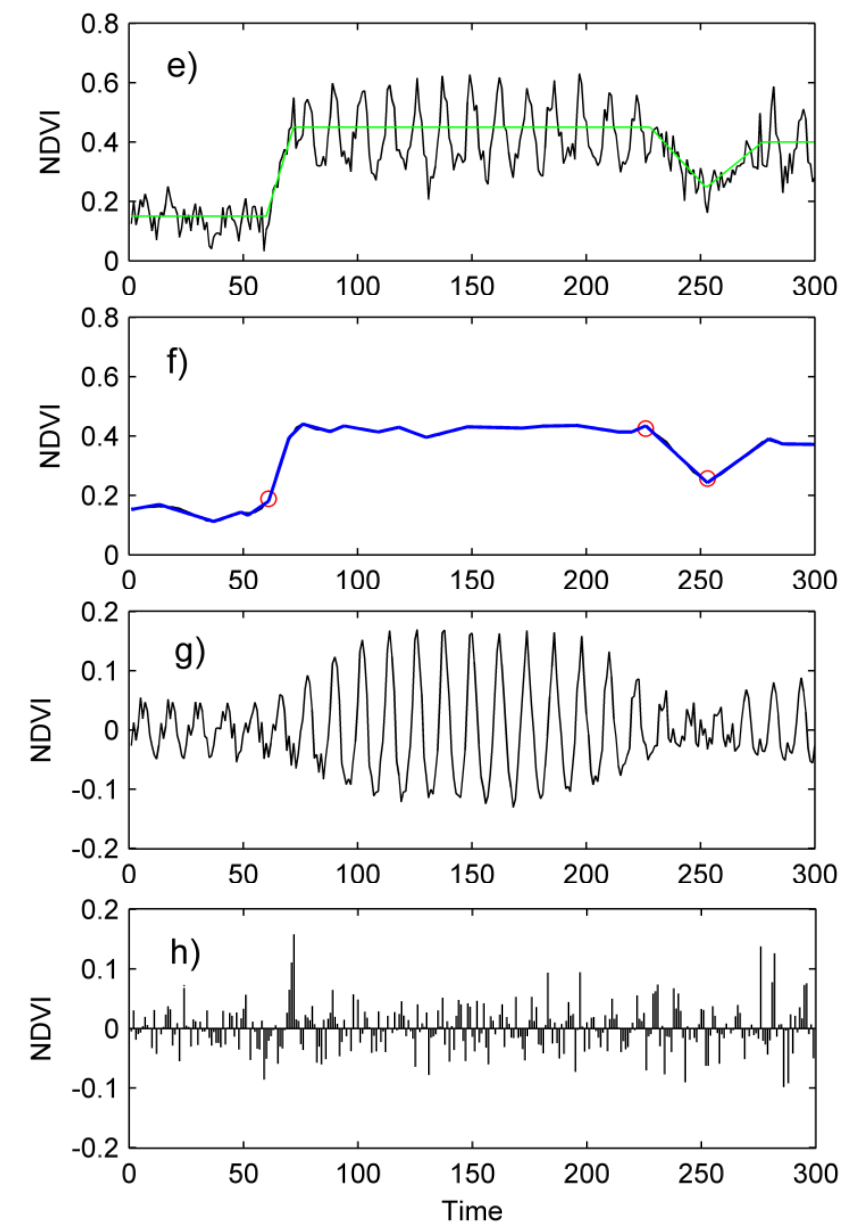

Fig. 5. The left- and right-hand columns show the results of DBEST's change detection algorithm for site 1and site 2 respectively. (a,e) - the simulated trends of NDVI at noise level $\sigma=0$ (in green) with simulated NDVI time series at noise level $\sigma=0.04$ (in black). (b,f) - decomposed trend components using STL (in black) and fit results obtained by DBEST (in blue) for detecting three major changes in the trend at each site. (c, g) - the decomposed seasonal, and (d, h) - remainder components. The detected breakpoints, corresponded to the three major changes, are marked with 
rings; a solid ring represents abrupt (level-shift) change, and an empty ring represents non-abrupt change. The simulated NDVI time series, shown for each site, is one of the 500 iterations of the simulation using a noise level of $\sigma=0.04$.
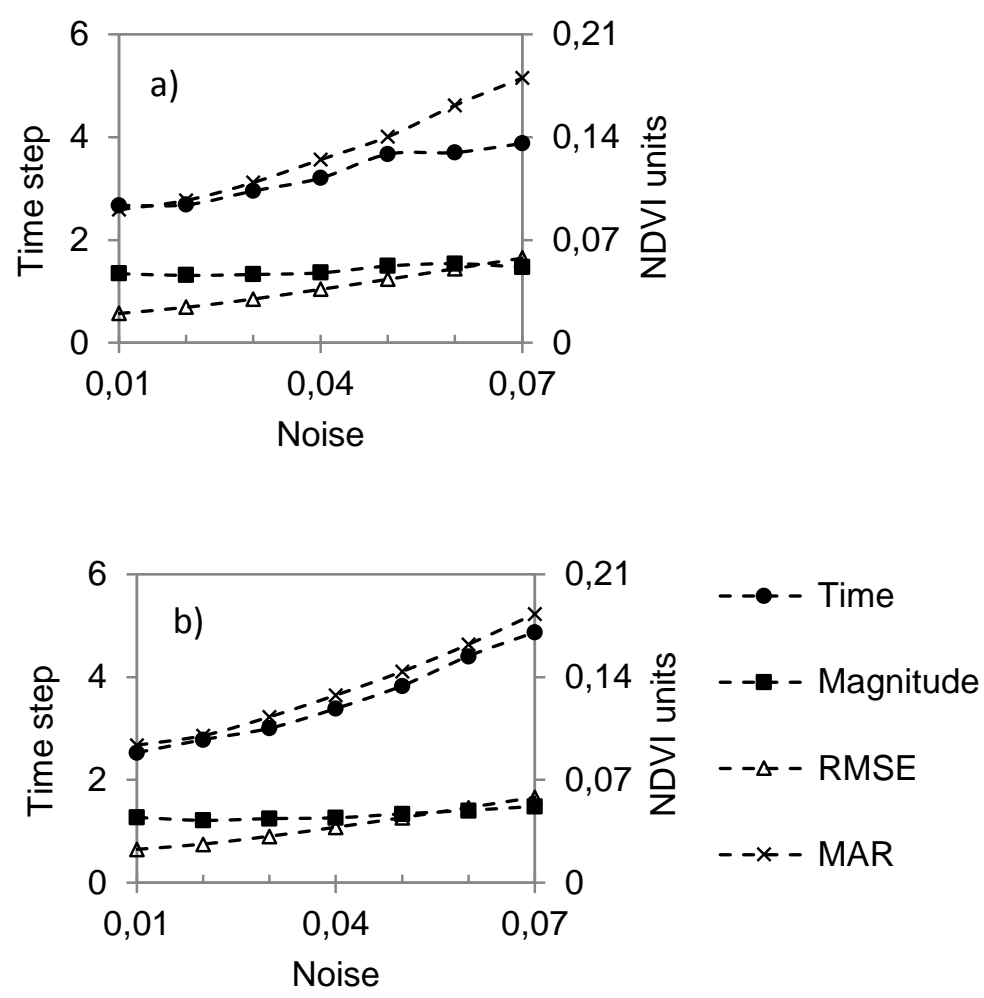

Fig. 6. Average RMSE for the time (left vertical axis), and magnitude (right vertical axis) estimates of three major changes within the simulated NDVI time series at (a) site 1 and (b) site 2. The average RMSE for the obtained fits, and average maximum-absolute-remainder (MAR) are also shown. The three major changes were detected by DBEST's change detection algorithm using the simulated NDVI time series with 500 iterations for different noise levels $(\sigma=0.01-0.07)$. 

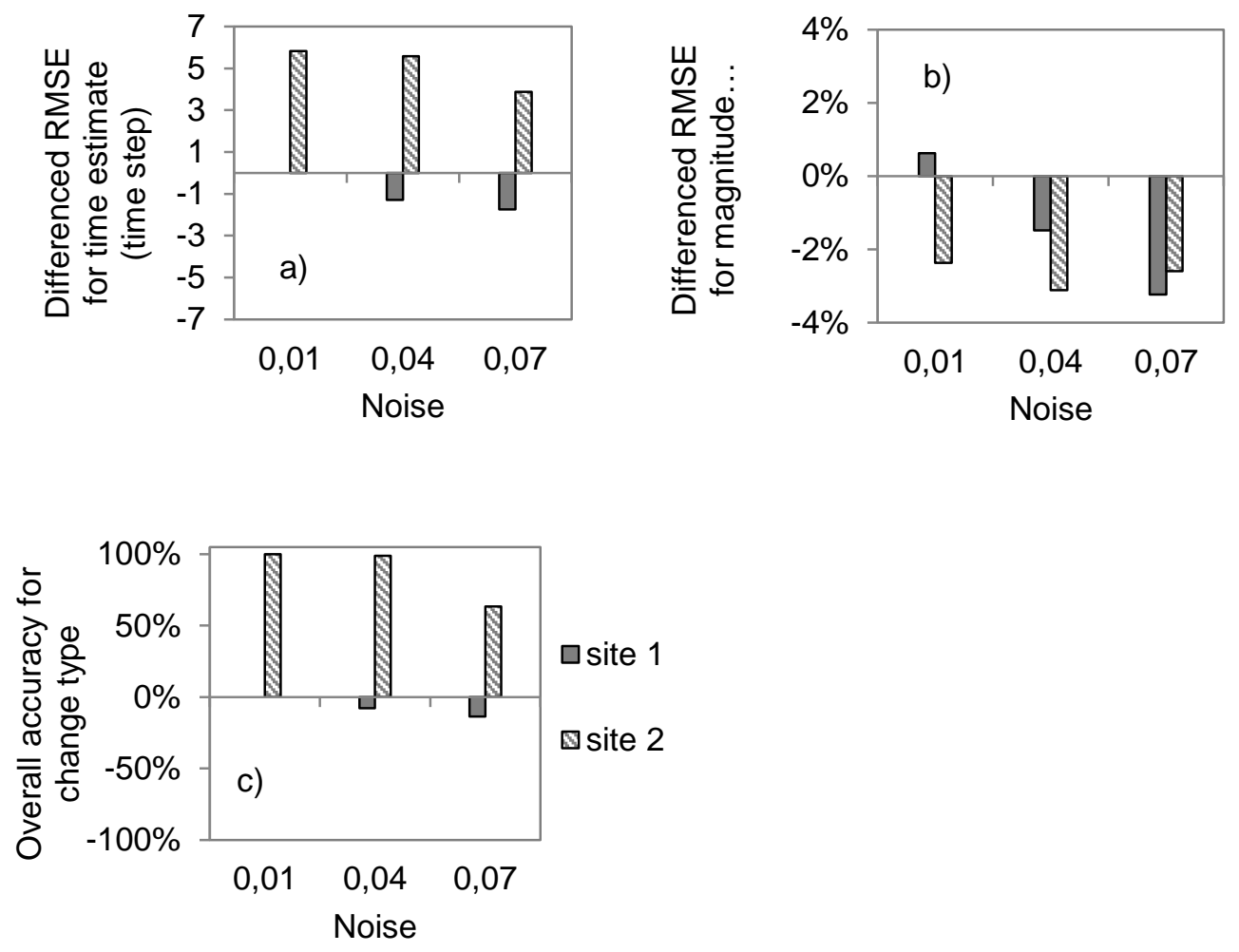

Fig. 7. Average RMSE, differenced between DBEST and BFAST, for (a) the time, and (b) magnitude estimates of one major change within the simulated NDVI time series at site 1 and site 2 . The major change was detected by both methods using the simulated NDVI time series with 500 iterations for each noise level $(\sigma=0.01,0.04,0.07)$. - (c) shows the overall accuracy of the change type, differenced between the methods. Positive values indicate better performance by DBEST than BFAST. 


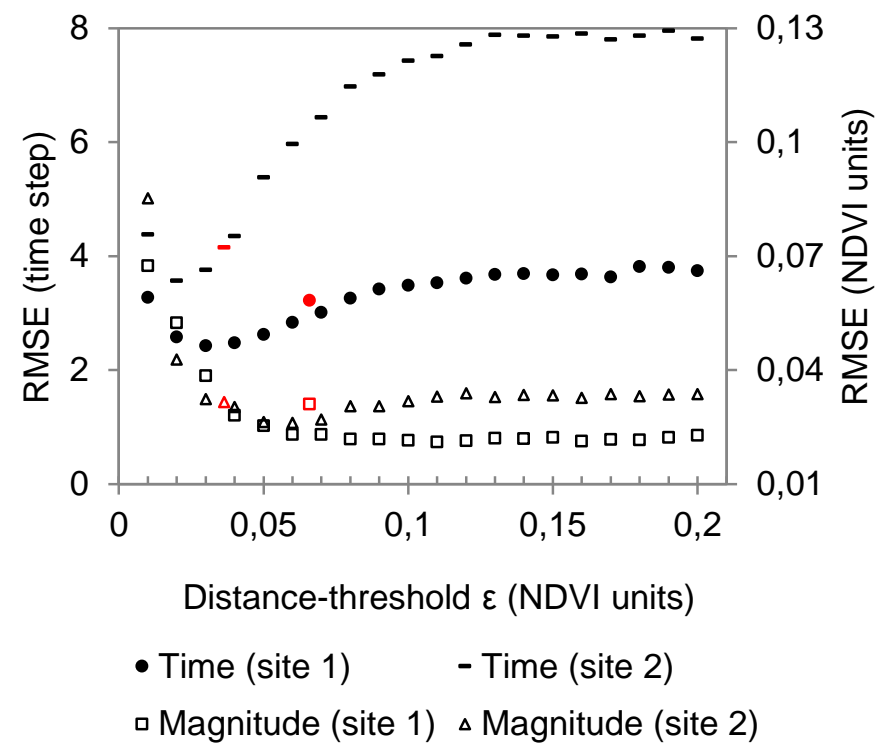

Fig. 8. Average RMSE for the estimated time (left vertical axis) and magnitude (right vertical axis) of three major changes found by DBEST's change detection algorithm for the simulated trend time series, as a function of the distance-threshold $(\varepsilon)$ (on horizontal axis). The input data consisted of 500 iterations with the noise level $\sigma=0.04$ at site 1 and site 2. Markers in red denote the average RMSEs obtained for time and magnitude estimates using the distance-threshold, computed by Eq. (7), in DBEST. 

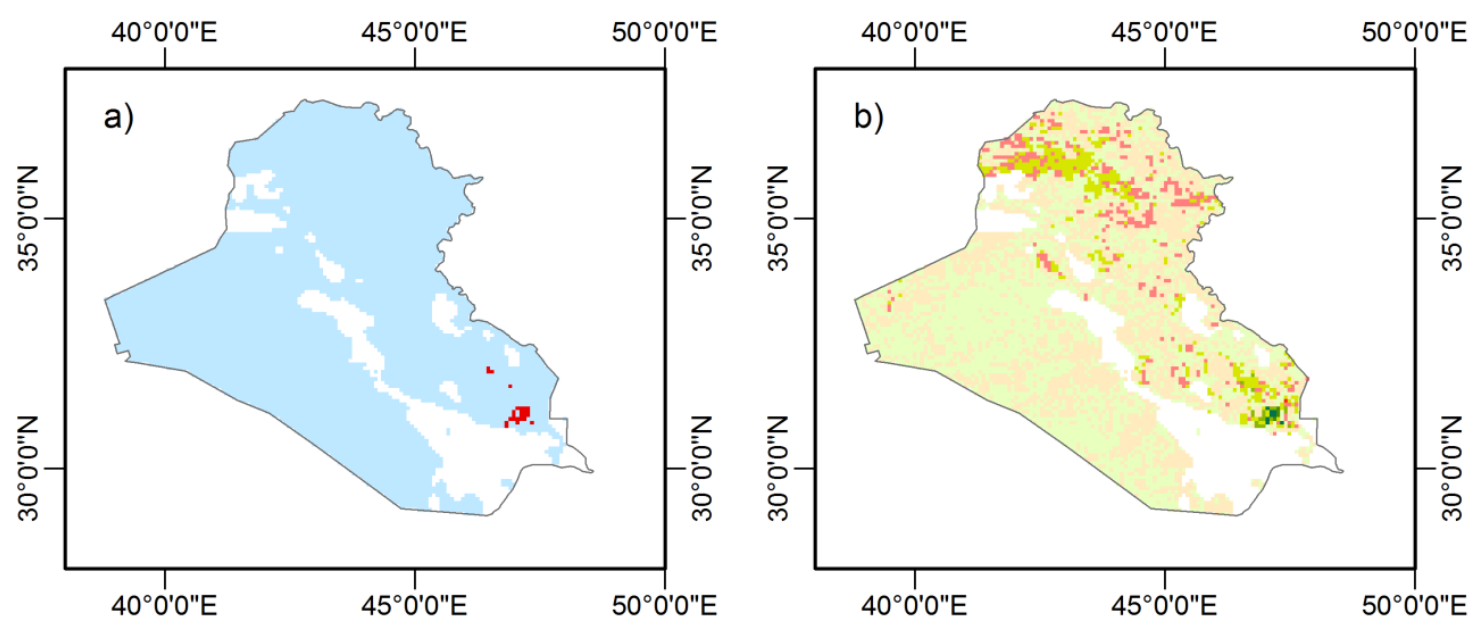

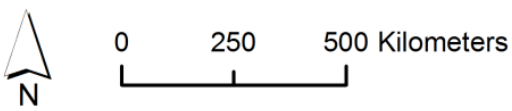

Change type

$\widehat{N}_{N} \quad \begin{array}{ccc}0 & 250 \quad 500 \text { Kilometers }\end{array}$

Major change in NDVI

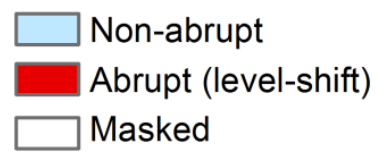

$\square-0.3--0.2 \square 0-0.1 \square 0.3-0.4$
$\square-0.2--0.1 \square 0.1-0.2 \square 0.4-0.5$
$\square-0.1-0 \quad \square \quad 0.2-0.3 \square$ Masked
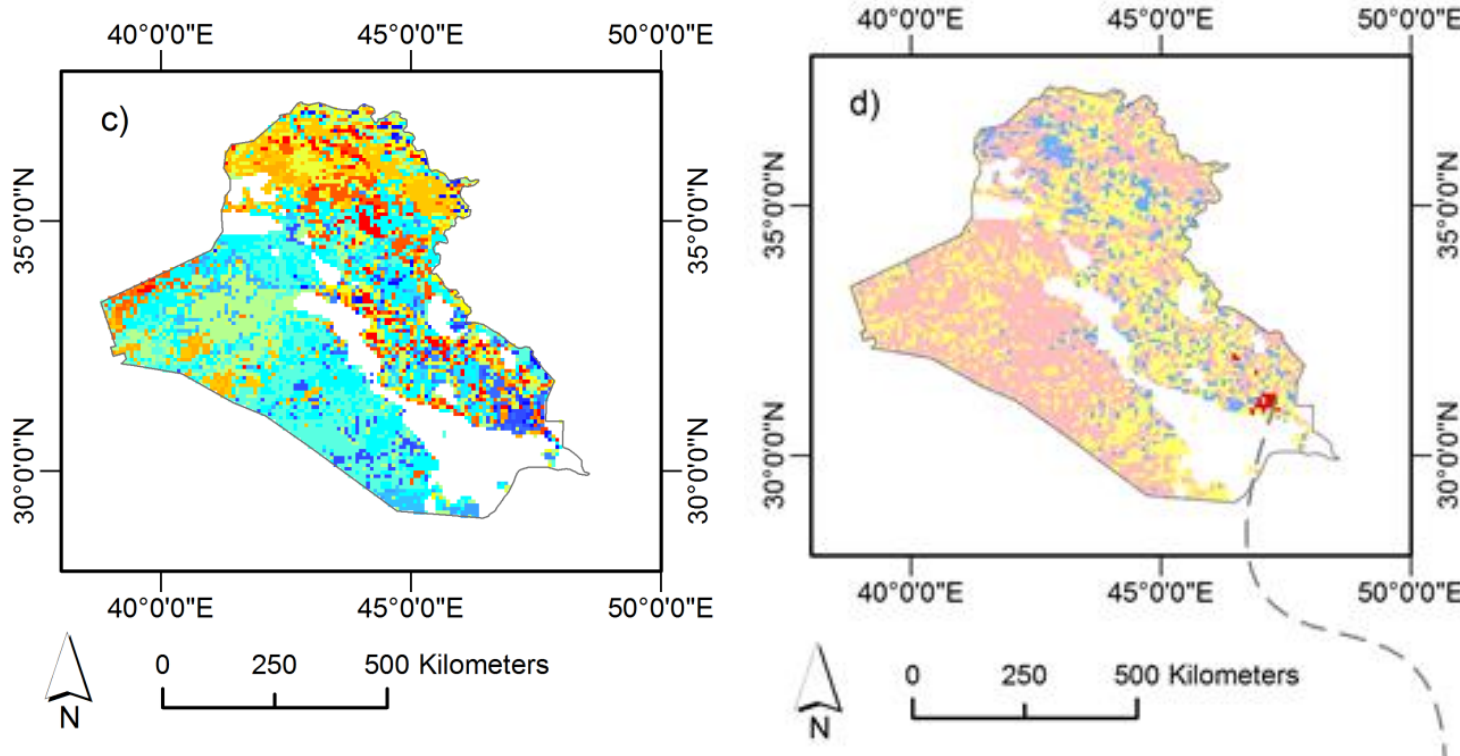

Start time (year)

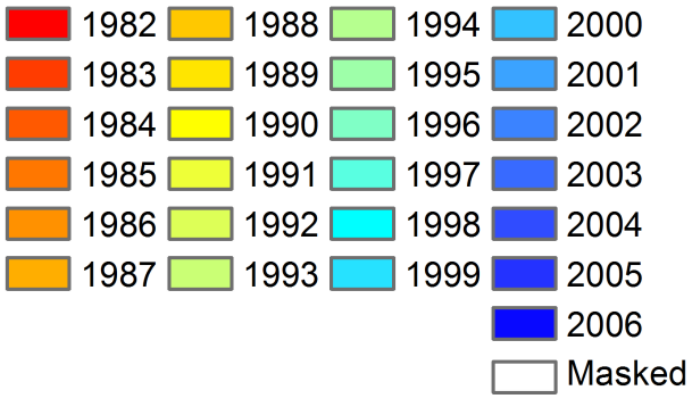

\section{Duration (month)}

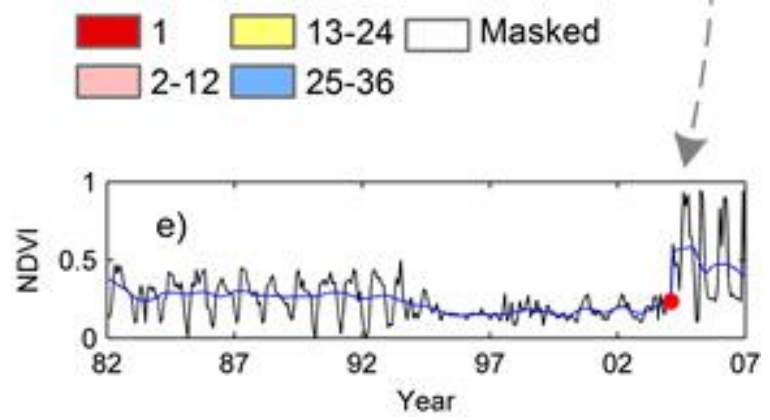


Fig. 9. DBEST estimated characteristics of major change in trend of monthly GIMMS-NDVI, 1982-2006, Iraq. The characteristics include: (a) change type, (b) change in NDVI, (c) start time, and (d) duration. Subfigure (e) represents the NDVI time series (in black), computed trend (in blue), and the detected major change (level-shift) (red dot) for one sample pixel located in areas with abrupt change ,as shown in subfigure (a), in southeast Iraq. Pixels with at least $75 \%$ valid data points (i.e., quality flag value zero) through the whole time span were analysed. Areas with a mean monthly NDVI $<0.1$ were masked out. All computations were done based on the monthly trend, but for an easier representation the start time is represented in years. 